\title{
Marion Peak Quadrangle, Fresno County, California- Analytic Data
}

\section{U.S. GEOLOGICAL SURVEY BULLETIN 1986}




\section{AVAILABILITY OF BOOKS AND MAPS OF THE U.S. GEOLOGICAL SURVEY}

Instructions on ordering publications of the U.S. Geological Survey, along with prices of the last offerings, are given in the current-year issues of the monthly catalog "New Publications of the U.S. Geological Survey." Prices of available U.S. Geological Survey publications released prior to the current year are listed in the most recent annual "Price and Availability List." Publications that are listed in various U.S. Geological Survey catalogs (see back inside cover) but not listed in the most recent annual "Price and Availability List" are no longer available.

Prices of reports released to the open files are given in the listing "U.S. Geological Survey Open-File Reports," updated monthly, which is for sale in microfiche from the U.S. Geological Survey, Books and Open-File Reports Section, Federal Center, Box 25425 , Denver, CO 80225 . Reports released through the NTIS may be obtained by writing to the National Technical Information Service, U.S. Department of Commerce, Springfield, VA 22161; please include NTIS report number with inquiry.

Order U.S. Geological Survey publications by mail or over the counter from the offices given below.

\section{BY MAIL}

\section{Books}

Professional Papers, Bulletins, Water-Supply Papers, Techniques of Water-Resources Investigations, Circulars, publications of general interest (such as leaflets, pamphlets, booklets), single copies of Earthquakes \& Volcanoes, Preliminary Determination of Epicenters, and some miscellaneous reports, including some of the foregoing series that have gone out of print at the Superintendent of Documents, are obtainable by mail from

\section{U.S. Geological Survey, Books and Open-File Reports Federal Center, Box 25425 Denver, CO 80225}

Subscriptions to periodicals (Earthquakes \& Volcanoes and Preliminary Determination of Epicenters) can be obtained ONLY from the

\section{Superintendent of Documents \\ Government Printing Omce \\ Washington, D.C. 20402}

(Check or money order must be payable to Superintendent of Documents.)

\section{Maps}

For maps, address mail orders to

\section{U.S. Geological Survey, Map Distribution \\ Federal Center, Box 25286 \\ Denver, CO 80225}

Residents of Alaska may order maps from

\author{
Alaska Distribution Section, U.S. Geological Survey, \\ New Federal Building - Box 12 \\ 101 Twelfth Ave., Fairbanks, AK 99701
}

\section{OVER THE COUNTER}

\section{Books}

Books of the U.S. Geological Survey are available over the counter at the following Geological Survey Public Inquiries Offices, all of which are authorized agents of the Superintendent of Documents:

- WASHINGTON, D.C.--Main Interior Bldg., 2600 corridor, 18 th and C Sts., NW.

- DENVER, Colorado--Federal Bldg., Rm. 169, 1961 Stout St.

- LOS ANGELES; California--Federal Bldg., Rm. 7638, 300 N. Los Angeles St.

- MENLO PARK, California--Bldg. 3 (Stop 533), Rm. 3128, 345 Middlefield Rd.

- RESTON, Virginia--503 National Center, Rm. 1C402, 12201 Sunrise Valley Dr.

- SALT LAKE CITY, Utah--Federal Bldg., Rm. 8105, 125 South State St.

- SAN FRANCISCO, California--Customhouse, Rm. 504, 555 Battery St.

- SPOKANE, Washington--U.S. Courthouse, Rm. 678, West 920 Riverside Ave..

- ANCHORAGE, Alaska--Rm. 101, 4230 University Dr.

- ANCHORAGE, Alaska--Federal Bldg, Rm. E-146, 701 C St.

\section{Maps}

Maps may be purchased over the counter at the U.S. Geological Survey offices where books are sold (all addresses in above list) and at the following Geological Survey offices:

- ROLLA, Missouri--1400 Independence Rd.

- DENVER, Colorado--Map Distribution, Bldg. 810, Federal Center

- FAIRBANKS, Alaska--New Federal Bldg., 101 Twelfth Ave. 


\section{Marion Peak Quadrangle,}

Fresno County, California-

Analytic Data

BY JAMES G. MOORE

U.S. GEOLOGICAL SURVEY BULLETIN 1986 


\section{U.S. DEPARTMENT OF THE INTERIOR}

MANUEL LUJAN, JR., Secretary

\section{U.S. GEOLOGICAL SURVEY}

Dallas L. Peck, Director

Any use of trade, product, or firm names

in this publication is for descriptive purposes only

and does not imply endorsement by the U.S. Government

For sale by the

Books and Open-File Reports Section

U.S. Geological Survey

Federal Center, Box 25425

Denver, CO 80225

\section{Library of Congress Cataloging-in-Publication Data}

Moore, James Gregory, 1930-

Marion Peak quadrangle, Fresno County, California, analytic data / by James

G. Moore

p. cm. - (U.S. Geological Survey bulletin ; 1986)

Includes bibliographical references

Supt. of Docs. no.: I 19:3:1986

1. Granite-California-Fresno County-Analysis. 2. Volcanic ash, tuff, etc.-California-Fresno County-Analysis. 3. Geology, Stratigraphic-

Cenozoic. 4. Geology-California-Fresno County. I. Title. II. Series. QE75.B9 no. 1986

[QE462.G7]

$557.3 \mathrm{~s}-\mathrm{dc} 20$

$91-15520$

[557.94'82] 


\section{CONTENTS}

Abstract 1
Introduction 1
General geology and age determinations 1

General geology and age determination 2
Sampling and analytic methods 2

Relation of $\mathrm{SiO}_{2}$ to specific gravity, color index, and magnetic susceptibility 2

Average granitic rock composition 3

References 3

FIGURES

1. Generalized geologic map of Marion Peak quadrangle (Moore, 1978) showing locations of analyzed samples 7

2. $\mathrm{SiO}_{2}$ variation diagrams for analyzed granitic rocks from the Marion Peak quadrangle 8

3. Classification scheme for granitic rocks from Streckeisen (1973) 9

4. Modal plots of samples from 15 of 22 granitic masses in the Marion Peak quadrangle; large symbols are averages 10

5. Relation of $\mathrm{SiO}_{2}$ content to volume percent mafic minerals and to specific gravity for about 200 analyzed granitic rocks in the Sierra Nevada between $37^{\circ}$ and $38^{\circ} \mathrm{N}$. latitude (Bateman and others, 1984) 12

6. Relation of $\mathrm{SiO}_{2}$ content to volume percent mafic minerals and to specific gravity for 15 analyzed granitic rocks in the Marion Peak quadrangle

7. Relation of magnetic susceptibility to volume percent of mafic minerals and to specific gravity for most of the 178 modally analyzed granitic rocks in the Marion Peak quadrangle (table 2) 13

\section{TABLES}

1. Chemical analyses and norms of granitic rocks 14

2. Modal analyses of granitic rocks 18

3. Chemical analyses and norms of Cenozoic volcanic rocks

4. Some features of mapped and sampled granitic units 23

5. Average chemical composition, in weight percent, of granitic rocks in the Marion Peak quadrangle 23 


\section{Marion Peak Quadrangle, Fresno County, California- Analytic Data}

\author{
By James G. Moore
}

\begin{abstract}
The Marion Peak 15-minute quadrangle includes about $620 \mathrm{~km}^{2}$ on the west slope of the Sierra Nevada in Fresno County, California, between $36^{\circ} 45^{\prime}$ and $37^{\circ} 00^{\prime} \mathrm{N}$. latitude and $118^{\circ} 30^{\prime}$ and $118^{\circ} 45 \mathrm{~W}$. longitude. This report supplements the geologic map of the Marion Peak quadrangle by providing modal and chemical analyses of the granitic and volcanic rock samples.

Granitic rocks of the batholith underlie 95 percent of the quadrangle area. Most of the remaining area is underlain by septa of pre-batholithic metamorphosed sediments and volcanic rocks which occur between the individual granitic masses. Cenozoic volcanic rocks underlie a small area, and they include an eroded dacitic volcanic center and scattered erosional remnants of basaltic lava flows.

About 300 samples of typical plutonic and volcanic rocks were collected during the course of geologic mapping; of these, 30 granitic, 2 metavolcanic, and 9 Cenozoic volcanic rocks were chemically analyzed for their major elements, and most were also analyzed for selected trace elements. In addition, 178 samples of granitic rocks were analyzed modally for the volume percent of their constituent minerals. Measurements of specific gravity and magnetic susceptibility are also included.

The average chemical composition of the granitic rocks in the quadrangle, estimated from the analyzed samples, is a silicic granodiorite that contains 69.2 weight percent $\mathrm{SiO}_{2}$.
\end{abstract}

\section{INTRODUCTION}

The Marion Peak quadrangle includes about $620 \mathrm{~km}^{2}$ on the west slope of the Sierra Nevada in Fresno County, California, between $36^{\circ} 45^{\prime}$ and $37^{\circ} 00^{\prime} \mathrm{N}$. latitude, and $118^{\circ} 30^{\prime}$ and $118^{\circ} 45^{\prime} \mathrm{W}$. longitude. The quadrangle lies chiefly between the Middle and South Forks of the Kings River and is largely within Kings Canyon National Park. The crest of Monarch Divide, the high ridge between these forks of the Kings River, culminates at Mt. Harrington $(11,005$ feet) near the west quadrangle boundary, and Marion Peak $(12,719$ feet) near the northeast corner of the

Manuscript approved for publication, March 25, 1990. quadrangle. Lowest elevations of the quadrangle are near the west boundary on the Middle Fork of the Kings River (4604 feet) and the South Fork (4138 feet).

Granitic rocks of the Sierra Nevada batholithic complex underlie about 95 percent of the area of the quadrangle. This report supplements the geologic map of the Marion Peak quadrangle (Moore, 1978) by providing modal and chemical analyses of the granitic and volcanic rock samples.

\section{GENERAL GEOLOGY AND AGE DETERMINATIONS}

The geology of the quadrangle is dominated by granitic intrusions composing the Sierra Nevada batholithic complex, which was emplaced largely in the Cretaceous. Granitic rocks of the batholith underlie 95 percent of the quadrangle area. Most of the remaining area is underlain by thin septa of pre-batholithic metamorphosed sedimentary and volcanic rocks which occur between the individual granitic masses. Cenozoic volcanic rocks underlie a small area; they include an eroded dacitic volcanic center and scattered erosional remnants of basaltic lava flows.

The oldest rocks of the quadrangle are small masses of generally silicic metavolcanic rocks preserved primarily as septa between individual granitic intrusive masses. In the northeast corner of the quadrangle, these metavolcanic rocks are closely associated with a mass of sheared granitic rocks cut by mafic dikes tentatively assigned to the Late Jurassic Independence dike swarm (Chen and Moore, 1979). The metavolcanic rocks in the northeast corner are part of the south end of the Mount Goddard pendant in which radiometric ages of 131-143 Ma indicate a Late Jurassic age (Tobish and others, 1986). Silicic metatuffaceous rocks on the west-central margin of the quadrangle are part of the Boyden Cave pendant, which has yielded a radiometric age of $106 \mathrm{Ma}$ (Saleeby and others, 1990). Hence the metamorphosed metavolcanic rocks of the quadrangle span some $37 \mathrm{~m} . y$. and apparently represent the volcanic cover associated with two general periods of plutonic intrusion that occurred in the Late Jurassic and the mid-Cretaceous. 
The Late Jurassic sequence of granitic rocks has not been dated within the quadrangle, but an age of $156 \mathrm{Ma}$ (Tobish and others, 1986) for a granitic unit within the Mount Goddard pendant cut by mafic dikes north of the quadrangle provides a basis for estimating the age of the sheared granitic unit of similar character in the northeast corner of the quadrangle.

Several masses of mid-Cretaceous hypabyssal and plutonic rocks associated with the metavolcanic rocks of the Boyden Cave pendant have been dated west of the quadrangle by $\mathrm{U} / \mathrm{Pb}$ zircon and $\mathrm{Rb} / \mathrm{Sr}$ bulk-rock isochron methods (Saleeby and others, 1990), which refine some of the earlier U/Pb age data of Chen and Moore (1982). Those that extend into the Marion Peak quadrangle are the schistose granodiorite of Tombstone Creek (102 Ma), metadacite porphyry of Boulder Creek (105 $\pm 3 \mathrm{Ma})$, granite of Grand Dike (103 $\pm 2 \mathrm{Ma}$ ), and granodiorite of Lightning Creek (100 Ma).

$\mathrm{K}-\mathrm{Ar}$ ages of samples collected outside the quadrangle that are a part of intrusive masses that extend into it include a biotite age of $81 \pm 3 \mathrm{Ma}$ from granodiorite of the Cartridge Pass pluton (Dodge and Moore, 1968) and biotite ages of $80.4 \mathrm{Ma}$ and a homblende age of $86.1 \mathrm{Ma}$ from the Paradise Granodiorite (Evernden and Kistler, 1970).

$\mathrm{U} / \mathrm{Pb}$ ages of samples of Cretaceous granitic plutons collected within the quadrangle include Lookout Peak (97 $\mathrm{Ma})$, North Dome (89?Ma), Paradise (83-86 Ma), and the heterogeneous granite of North Mountain, which has failed to yield a concordant age (Chen and Moore, 1982).

Cenozoic volcanic rocks within the quadrangle include the eroded Windy Peak dacitic volcanic neck and associated lava flows in the canyon of the Middle Fork of the Kings River, and small, flow remnants of potassic basaltic composition that are widely scattered over the western part of the quadrangle. Biotite and hornblende from the Windy Peak dacitic volcanic neck have been dated by the K-Ar method at $4.4 \pm 0.4 \mathrm{Ma}$, and $4.5 \pm 0.4 \mathrm{Ma}$, respectively; a pebble of dacite from the Kings River fan, apparently derived from the volcanic neck, yielded a whole rock age of $4.03 \pm 0.12 \mathrm{Ma}$ (Huber, 1981).

The basaltic remnants within the quadrangle have not been dated. A basalt flow remnant $15 \mathrm{~km}$ west of the quadrangle on Rancheria Creek yielded a whole rock K-Ar age of $3.20 \pm 0.06 \mathrm{Ma}$ and the basalt flow of Stony Flat 15 $\mathrm{km}$ west-southwest of the quadrangle yielded a whole rock $\mathrm{K}$-Ar age of 3.40 $\pm 0.06 \mathrm{Ma}$ (Huber, 1981).

\section{SAMPLING AND ANALYTIC METHODS}

About 300 samples of typical plutonic and volcanic rocks were collected during the course of mapping within the quadrangle. Of these, 30 granitic, 2 metavolcanic, and 9 Cenozoic volcanic rocks were chemically analyzed for their major elements, and most were also analyzed for selected trace elements (tables 1 and 3). Older chemical analyses were performed by the rapid method of Shapiro and Brannock (1962). All other analyses were made by standard $\mathrm{X}$-ray fluorescence techniques with $\mathrm{Na}_{2} \mathrm{O}$, and in some cases $\mathrm{K}_{2} \mathrm{O}$, determined by flame photometry. Ferrous iron, $\mathrm{CO}_{2}, \mathrm{H}_{2} \mathrm{O}^{+}$, and $\mathrm{H}_{2} \mathrm{O}^{-}$were determined by standard wet chemical methods. The generally regular relation of $\mathrm{SiO}_{2}$ to the other major elements in the analyzed granitic rocks is shown in the variation diagrams of figure 2 .

Altogether, 178 samples of granitic rocks were analyzed modally for the volume percent of their constituent minerals (table 2). Modal analyses were made by counting over 1,000 points on stained rock slabs (Norman, 1974). The color differences provide a basis for identifying and measuring the volume percent of quartz, plagioclase, K-feldspar, and mafic minerals (chiefly biotite, hornblende, sphene, and magnetite.

The locations of analyzed samples are shown in figure 1 , and the modal proportions of quartz, K-feldspar, and plagioclase in the granitic rocks appear in the triangular plots of figure 4 , which provide the basis for naming the rocks according to the classification (fig. 3) of the International Union of Geological Sciences (Streckeisen, 1973).

Specific gravity was determined by weighing hand samples in air and water on a beam balance. Magnetic susceptibility was measured on 1 kilogram hand samples with a Geoinstruments JH-5 susceptibility meter. Direct readings in SI units $\times 10^{-5}$ were doubled in accordance with the procedures used for hand samples.

\section{RELATION OF $\mathrm{SIO}_{2}$ TO SPECIFIC GRAVITY, COLOR INDEX, AND MAGNETIC SUSCEPTIBILITY}

The relation between major element chemical constituents and other parameters that can be measured more easily and at less cost can lead toward a better appraisal of compositional variation of the diverse plutonic rocks in a given area. Examination of about 200 analyses of granitic rock samples collected between $37^{\circ}$ and $38^{\circ}$ N. latitude in the Sierra Nevada (Bateman and others, 1984) indicates that the bulk specific gravity of hand specimens and volume percent of mafic minerals (color index) both correlate relatively well with each other and inversely with the weight percent of $\mathrm{SiO}_{2}$ (fig. 5). The inverse correlation of bulk specific gravity with weight percent $\mathrm{SiO}_{2}$ is particularly good $\left(\mathrm{R}^{2}=0.820\right)$, such that $\mathrm{SiO}_{2}=317.93$-(sp. gr./0.010752). The analyzed granitic rocks within the quadrangle show these same general trends, but scatter is greater because of limited analyses (fig. 6). 
Measurements of magnetic susceptibility show a direct relation to both specific gravity and volume percent of mafic minerals measured on the same samples (fig. 7).

\section{AVERAGE GRANITIC ROCK COMPOSITION}

The estimation of the average chemical composition of the granitic rocks in the quadrangle is useful for comparison with other areas, and ultimately for development of models for the genesis of the batholith. Because of its ease of measurement, specific gravity is generally obtained for a higher proportion of available samples than are other analytical procedures. An estimate of the average specific gravity of each pluton is derived from a weighted average of the determinations made on samples from that pluton. Then the average specific gravity of the plutonic rocks in the entire quadrangle is determined by considering the area of each mapped plutonic mass (table 4). From this grand average specific gravity, the average $\mathrm{SiO}_{2}$ content of 69.2 weight percent is determined from the overall relation of specific gravity to $\mathrm{SiO}_{2}$. Finally other major element concentrations are estimated for this average $\mathrm{SiO}_{2}$ content from variation diagrams for all analyzed rocks in the quadrangle, thus establishing the overall estimate of the average plutonic rock in the quadrangle.

The 69.2 percent $\mathrm{SiO}_{2}$ average for granitic rocks of the Marion Peak quadrangle is somewhat less than the 70.5 percent estimated for the Mt. Whitney quadrangle adjacent on the southeast (Moore, 1987), and more than the 68.2 percent estimated for the Mt. Pinchot quadrangle adjacent to the east (Moore, 1963). The estimated average composition of Mesozoic plutonic rocks of the Sierra Nevada is 68.4 percent (Dodge, 1972).

\section{REFERENCES}

Bateman, P.C., Dodge, F.C.W., and Bruggman, P.E., 1984, Major oxide analyses, CIPW norms, modes, and bulk specific gravities of plutonic rocks from the Mariposa $1^{\circ} \times 2^{\circ}$ sheet, central Sierra Nevada, California: U.S. Geological Survey Open-File Report 84-162, 50 p.

Chen, J.H., and Moore, J.G., 1979, Late Jurassic Independence dike swarm in eastern California: Geology, v. 7, no. 3, p. 129-133.
Chen, J.H., and Moore, J.G., 1982, Uranium-lead isotopic ages from the Sierra Nevada batholith, California: Journal of Geophysical Research, v. 87, p. 4761-4784.

Dodge, F.C.W., 1972, Trace-element contents of some plutonic rocks of the Sierra Nevada batholith: U.S. Geological Survey Bulletin 1314-F, 13 p.

Dodge, F.C.W. and Moore, J.G., 1968, Occurrence and composition of biotites from the Cartridge Pass pluton of the Sierra Nevada batholith, California in Geological Survey Research 1968: U.S. Geological Survey Professional Paper 600-B, p. 6-10.

Evernden, J.H. and Kistler, R.W., 1970, Chronology of emplacement of Mesozoic batholithic complexes in California and western Nevada: U.S. Geological Survey Professional Paper 623, 42 p.

Huber, N.K., 1981, Amount and timing of late Cenozoic uplift and tilt of the central Sierra Nevada, California-Evidence from the upper San Joaquin River basin: U.S. Geological Survey Professional Paper 1197, 28 p.

Moore, J.G., 1963, Geology of the Mount Pinchot quadrangle, southern Sierra Nevada, California: U. S. Geological Survey Bulletin 1130, $152 \mathrm{p}$.

1978, Geologic map of the Marion Peak quadrangle, Sierra Nevada, California: U.S. Geological Survey Geologic Quadrangle Map GQ 1399.

1987, Geologic map of the Mount Whitney quadrangle, Inyo and Tulare counties, California: U.S. Geological Survey Geologic Quadrangle Map GQ 1545.

Norman, M.B., 1974, Improved techniques for selective staining of feldspar and other minerals using amaranth: U.S. Geological Survey Journal of Research, v. 2, p. 7379.

Saleeby, J.B., Kistler, R.W., Longiaru, S., Moore, J.G., and Nokleberg, W.J., 1990, Middle Cretaceous silicic metavolcanic rocks in the Kings Canyon area, central Sierra Nevada, California, in Anderson, J.L., ed., The nature and origin of Cordilleran magmatism: Boulder, Colorado, Geological Society of America Memoir 174, p. 251-270.

Shapiro, L., and Brannock, W.W., 1962, Rapid analyses of silicate, carbonate, and phosphate rocks: U.S. Geological Survey Bulletin 1144A, $56 \mathrm{p}$.

Streckeisen, A.L., 1973, Plutonic rocks, classification and nomenclature recommended by the International Union of Geological Sciences Subcommission on the Systematics of Igneous Rocks: Geotimes, v. 18, no. 10, p. 26-30.

Tobish, O.T., Saleeby, J.B., and Fiske, R.S., 1986, Structural history of continental volcanic arc rocks, eastern Sierra Nevada, California: a case for extensional tectonics: Tectonics, v. 5, p. 65-94. 

FIGURES 1-7; TABLES 1-5 



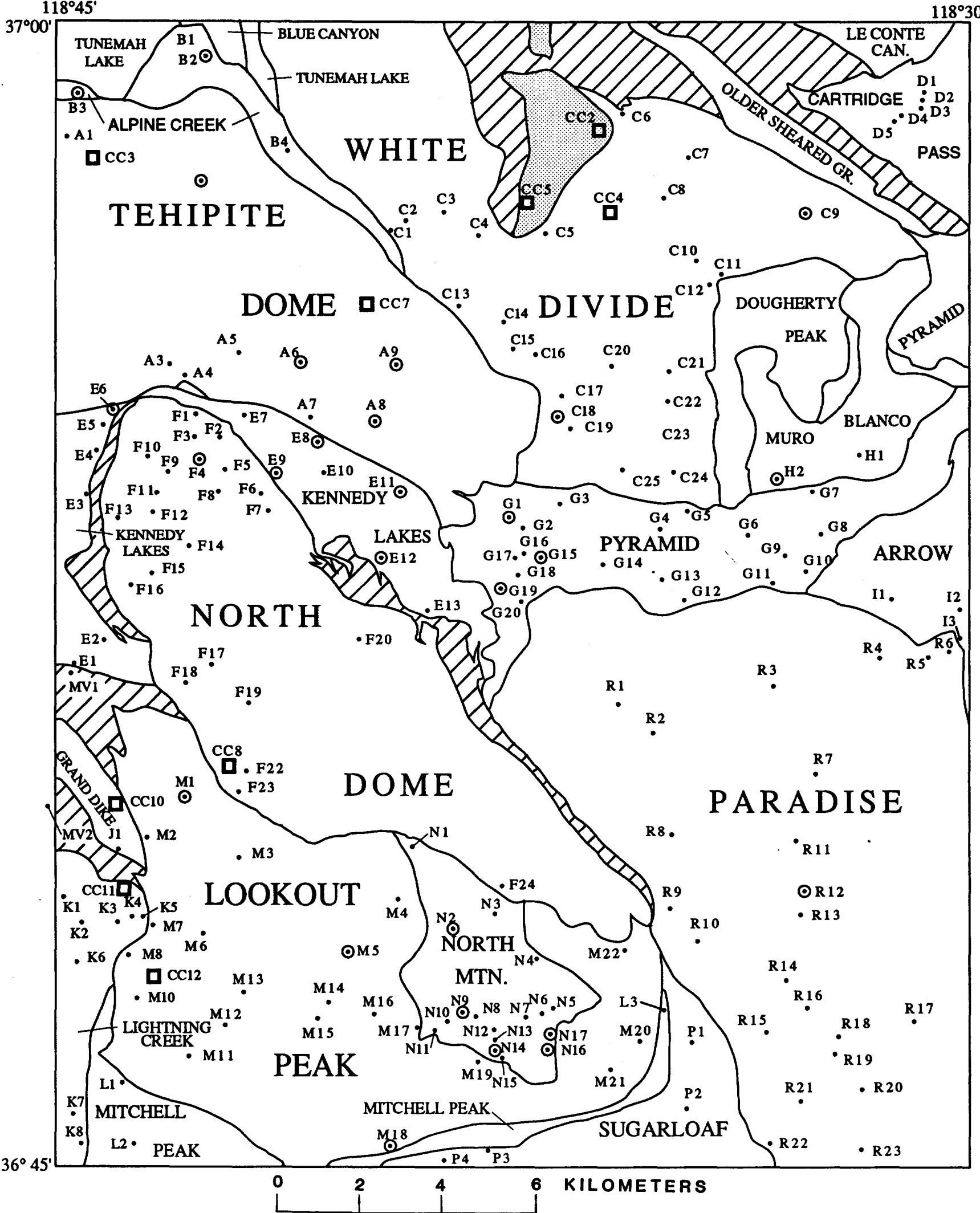

Figure 1. Generalized geologic map of Marion Peak quadrangle (Moore, 1978) showing locations of analyzed samples. Plutonic rocks shown by circles (chemically analyzed, table 1) and dots (modally analyzed, table 2). Chemically analyzed Cenozoic volcanic rocks shown by squares. Mesozoic granitic rocks are unpatterned, Cenozoic volcanic rocks have a shaded pattern, and metamorphic rocks have a lined pattern. Names of plutonic rock units shown. 

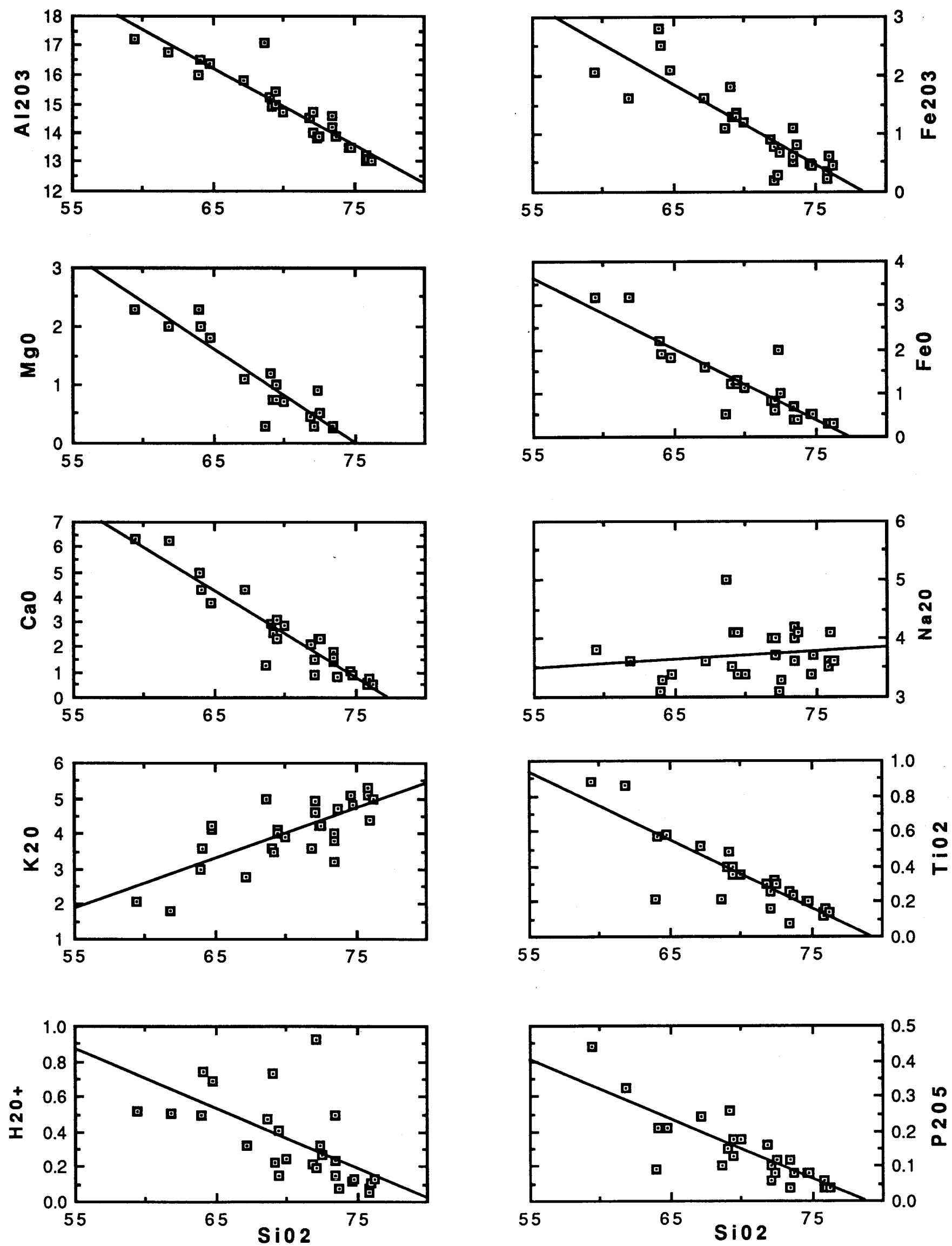

Figure 2. $\mathrm{SiO}_{2}$ variation diagrams for analyzed granitic rocks from the Marion Peak quadrangle. Least squares regression lines through data were used to estimate the average major elements based on the $\mathrm{SiO}_{2}$ average of 69.2 weight percent (table 5). 


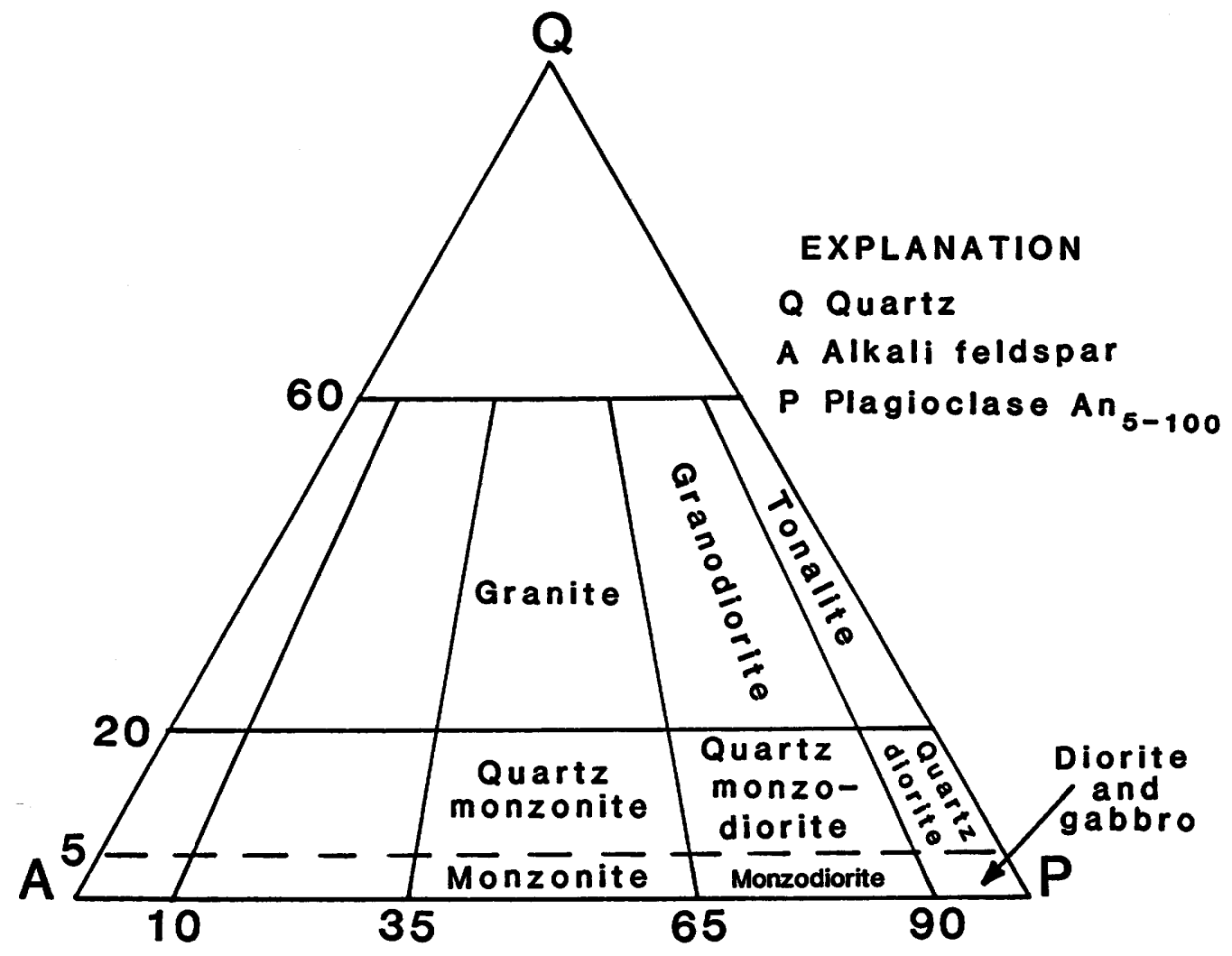

Figure 3. Classification scheme for granitic rocks from Streckeisen (1973). 

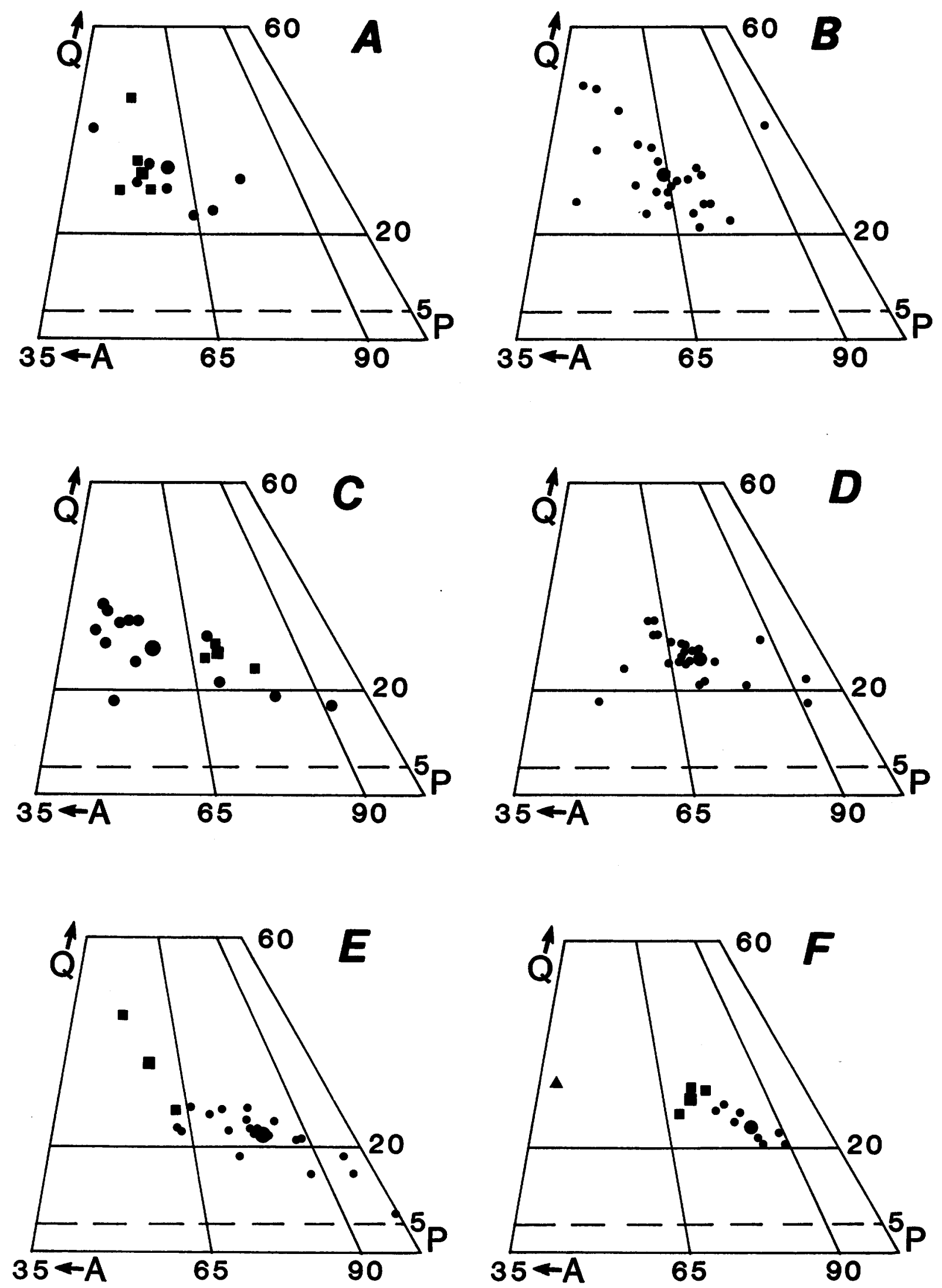

Figure 4. Modal plots of samples from 15 of 22 granitic masses in the Marion Peak quadrangle; large symbols White Divide. C, Granite of Kennedy Lakes (circles) and Cartridge Pass pluton (squares). $D$, Granodiorite of North (circles), Arrow pluton (squares), and Granite of Grand dike (triangle). G, Granodiorite of Mitchell Peak (circles) pluton. $K$, Averages of analyzed granitic masses. See figure 3 for classification scheme. 

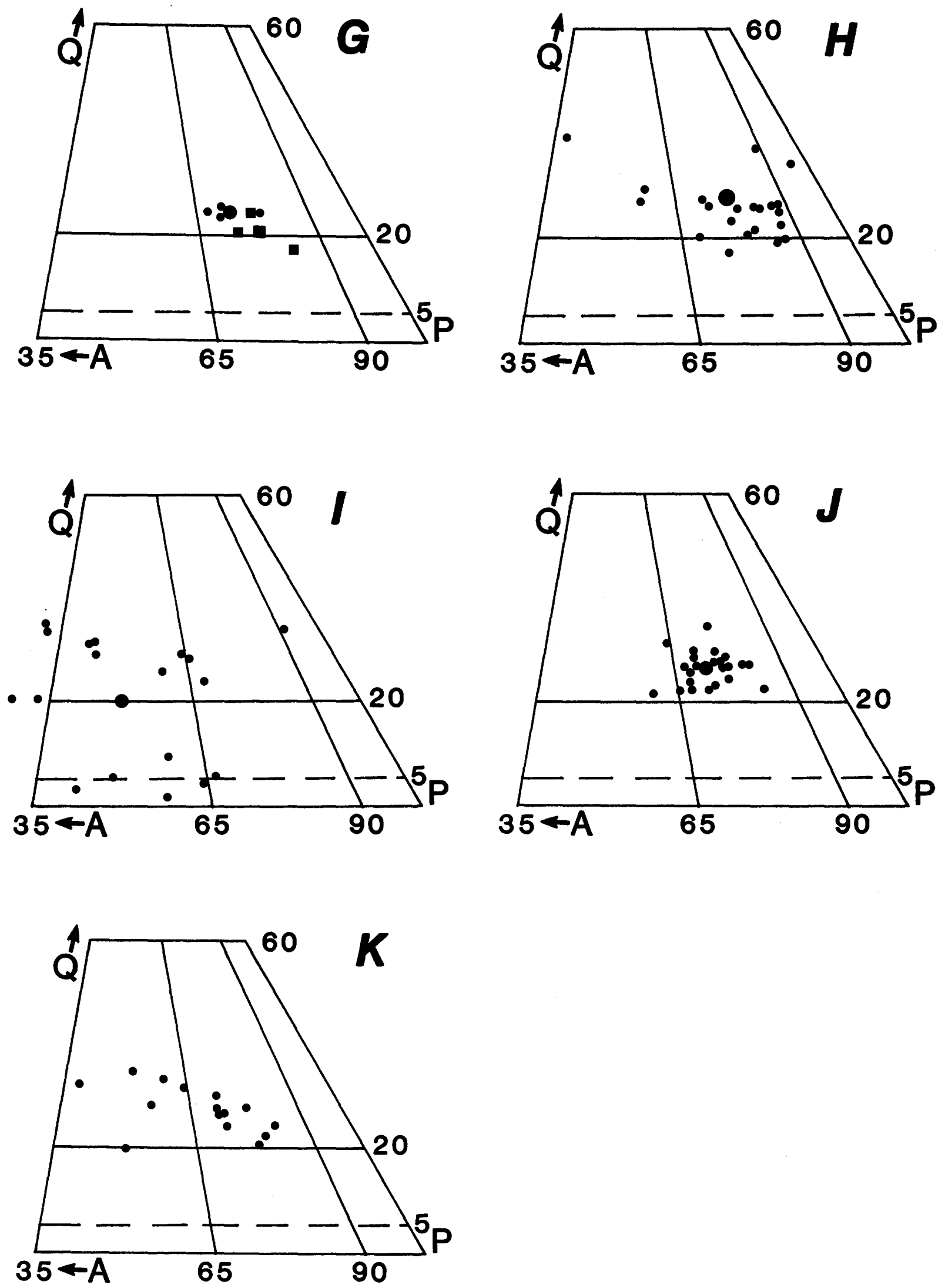

are averages. A, Granite of Tehipite Dome (circles) and Granite of Alpine Creek (squares). B, Granodiorite of Dome. E, Pyramid pluton (circles) and Granodiorite of Muro Blanco (squares). $F$, Granodiorite of Lightning Creek and Granodiorite of Sugarloaf (squares). H, Granodiorite of Lookout Peak. I, Granite of North Mountain. J, Paradise 

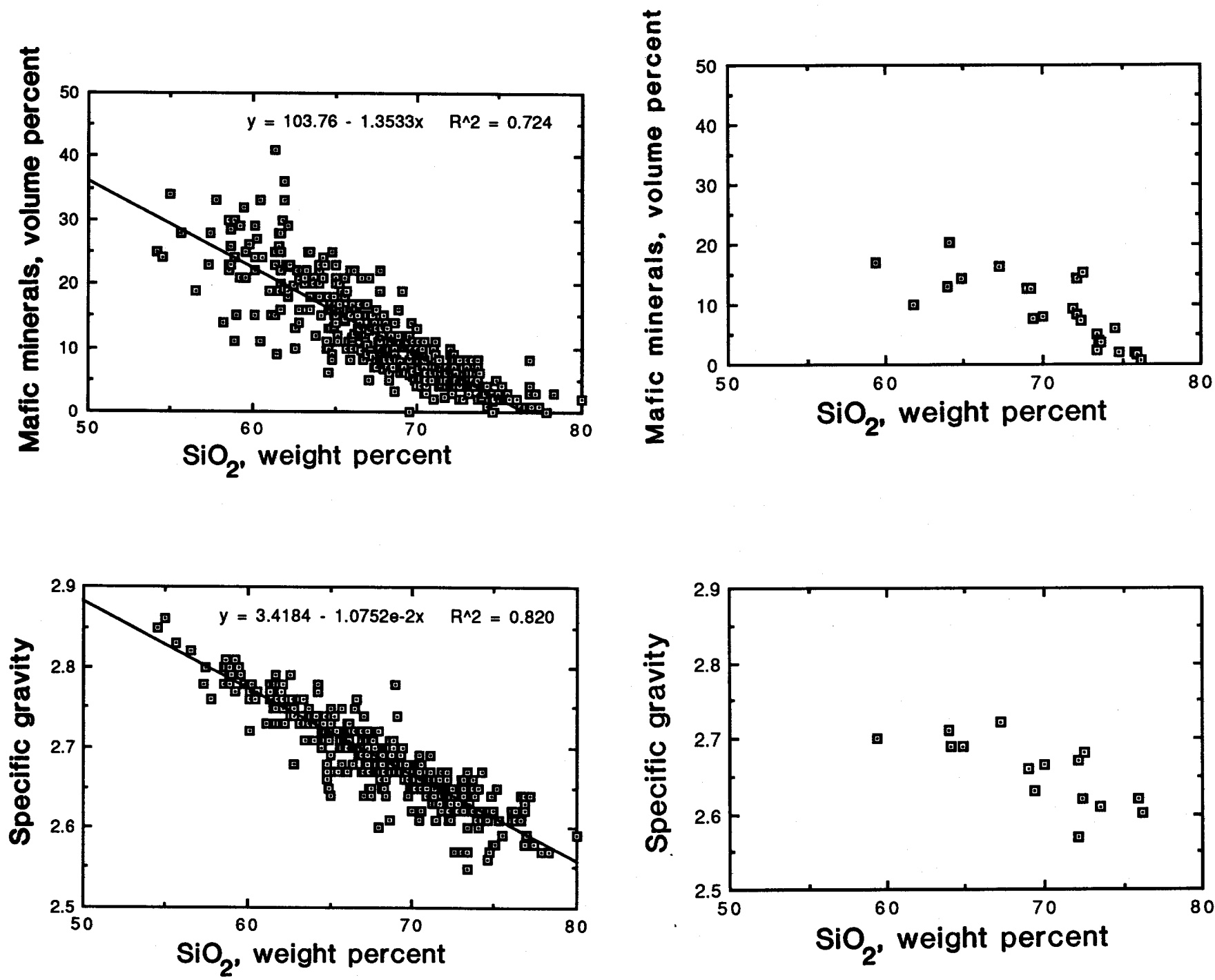

Figure 5. Relation of $\mathrm{SiO}_{2}$ content to volume percent mafic minerals and to specific gravity for about 200 analyzed granitic rocks in the Sierra Nevada between $37^{\circ}$ and $38^{\circ}$ $N$. latitude (Bateman and others, 1984). Equations for least

Figure 6. Relation of $\mathrm{SiO}_{2}$ content to volume percent mafic minerals and to specific gravity for 15 analyzed granitic rocks in the Marion Peak quadrangle. 

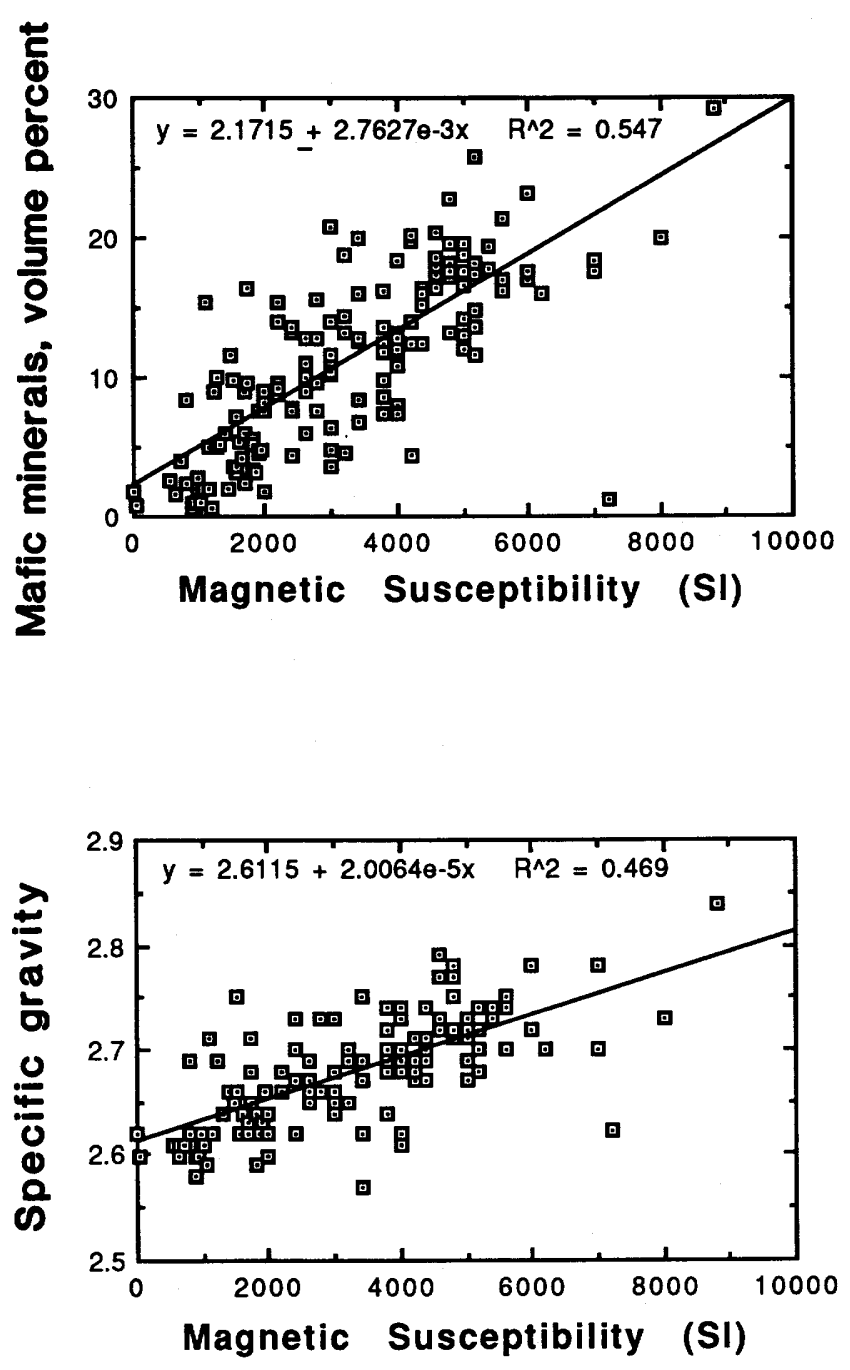

Figure 7. Relation of magnetic susceptibility to volume percent of mafic minerals and to specific gravity for most of the 178 modally analyzed granitic rocks in the Marion Peak quadrangle (table 2). Magnetic susceptibility is reported in dimensionless $\mathrm{SI}$ units $\times 10^{-5}$. Equations for least squares regression lines through data are shown. 
Table 1. Chemical analyses and norms of granitic rocks

[ $\dagger$, Analysis performed in the rapid rock analysis laboratory under the supervision of Leonard Shapiro; analysts: P. Elmore, S. Botts, G. Chloe, H. Smith, J. Kelsey, L. Artis and J. Glenn. *, Analysis by X-ray spectroscopy; analysts D. Vivit, M. Dyslin. Analysis of $\mathrm{FeO}_{2} \mathrm{H}_{2} \mathrm{O}$, and $\mathrm{CO}_{2}$ by chemical methods; analyst, $N$. Elsheimer. -, no data]

\begin{tabular}{|c|c|c|c|c|c|c|c|c|c|c|c|c|}
\hline \multirow[b]{2}{*}{$\begin{array}{l}\text { Field No. } \\
\text { Map No. }\end{array}$} & \multicolumn{3}{|c|}{ Granite of Tehipite Dome } & \multicolumn{2}{|c|}{$\begin{array}{c}\text { Granite of } \\
\text { Alpine Creek }\end{array}$} & \multicolumn{4}{|c|}{ Granodiorite of White Divide } & \multicolumn{3}{|c|}{ Granite of Kennedy Lakes } \\
\hline & $\begin{array}{c}67 \mathrm{M} 24+ \\
\mathrm{A} 6\end{array}$ & $\begin{array}{c}68 \mathrm{M} 50^{*} \\
\mathrm{A8}\end{array}$ & $\begin{array}{l}\text { 68M51* } \\
\text { A9 (dior.) }\end{array}$ & $\begin{array}{c}\text { M735* } \\
\text { B2 }\end{array}$ & $\begin{array}{l}\text { M742* } \\
\text { B3 }\end{array}$ & $\begin{array}{l}64 \mathrm{M} 20^{*} \\
\mathrm{C} 9\end{array}$ & $\begin{array}{c}\text { 64M37* } \\
\text { C18 }\end{array}$ & $\begin{array}{c}64 \mathrm{Mg} 9+ \\
\mathrm{C} 23\end{array}$ & $\begin{array}{l}\text { 64M10* } \\
\text { C25 }\end{array}$ & $\begin{array}{c}67 \mathrm{M} 40^{*} \\
\mathrm{E} 6\end{array}$ & $\begin{array}{c}67 \mathrm{M} 43+ \\
\text { E9 }\end{array}$ & $\begin{array}{c}67 \mathrm{M} 46^{*} \\
\mathrm{E8}\end{array}$ \\
\hline \multicolumn{13}{|c|}{ Chemical analyses (weight percent) } \\
\hline $\mathrm{SiO}_{2}$ & 72.4 & 72.5 & 61.8 & 73.4 & 74.6 & 69.2 & 73.4 & 69.5 & 71.9 & 72.1 & 68.7 & 76.0 \\
\hline $\mathrm{Al}_{2} \mathrm{O}_{3}$ & 13.8 & 13.9 & 16.8 & 14.2 & 13.5 & 14.9 & 14.2 & 15.4 & 14.5 & 14.0 & 17.1 & 13.1 \\
\hline $\mathrm{Fe}_{2}^{2} \mathrm{O}_{3}^{3}$ & 0.30 & 0.67 & 1.60 & 0.51 & 0.48 & 1.28 & 0.62 & 1.30 & 0.92 & 0.20 & 1.10 & 0.60 \\
\hline $\mathrm{FeO}^{2}$ & 2.00 & 0.97 & 3.20 & 0.70 & 0.49 & 1.60 & 0.70 & 1.30 & 0.83 & 0.78 & 0.48 & 0.26 \\
\hline $\mathrm{MgO}$ & 0.90 & 0.50 & 2.00 & 0.25 & $<0.2$ & 0.75 & 0.25 & 1.00 & 0.45 & $<0.2$ & 0.28 & $<0.2$ \\
\hline $\mathrm{CaO}$ & 2.30 & 2.34 & 6.24 & 1.42 & 1.06 & 2.54 & 1.80 & 2.30 & 2.08 & 0.90 & 1.30 & 0.72 \\
\hline $\mathrm{Na}_{2} \mathrm{O}$ & 3.10 & 3.30 & 3.60 & 4.00 & 3.40 & 4.10 & 4.20 & 4.10 & 4.00 & 3.70 & 5.00 & 4.10 \\
\hline $\mathrm{K}_{2} \mathrm{O}^{2}$ & 4.20 & 4.16 & 1.82 & 4.04 & 5.06 & 3.50 & 3.22 & 4.00 & 3.62 & 4.88 & 5.00 & 4.38 \\
\hline $\mathrm{H}_{2}^{2} \mathrm{O}^{+}$ & 0.32 & 0.27 & 0.51 & 0.15 & 0.12 & 0.23 & 0.24 & 0.41 & 0.22 & 0.92 & 0.47 & 0.11 \\
\hline $\mathrm{H}_{2}^{2} \mathrm{O}^{-}$ & 0.14 & 0.11 & 0.12 & 0.10 & 0.05 & 0.06 & 0.11 & 0.10 & 0.03 & 0.20 & 0.09 & 0.04 \\
\hline $\mathrm{TiO}_{2}^{2}$ & 0.32 & 0.30 & 0.86 & 1.20 & 0.20 & 0.48 & 0.26 & 0.36 & 0.30 & 0.16 & 0.22 & 0.16 \\
\hline $\mathrm{P}_{2} \mathrm{O}_{5}^{2}$ & 0.08 & 0.12 & 0.32 & 0.15 & 0.08 & 0.26 & 0.12 & 0.13 & 0.16 & 0.06 & 0.10 & 0.04 \\
\hline MnO & 0.08 & 0.07 & 0.10 & 1.40 & 0.04 & 0.09 & 0.05 & 0.12 & 0.07 & 0.04 & 0.09 & 0.04 \\
\hline LOI & - & 0.42 & 0.43 & - & 0.26 & 0.22 & 0.37 & - & 0.22 & 2.14 & - & 0.17 \\
\hline $\mathrm{CO}_{2}$ & $<.05$ & - & - & $<.05$ & - & - & - & $<.05$ & - & - & $<.05$ & - \\
\hline Total & 99.9 & 99.6 & 99.4 & 99.9 & 99.5 & 99.2 & 99.5 & 100.0 & 99.3 & 100.3 & 99.9 & 99.9 \\
\hline
\end{tabular}

Trace elements (parts per million)

\begin{tabular}{|c|c|c|c|c|c|c|c|c|c|c|c|c|}
\hline $\mathrm{Ba}$ & 700 & 630 & 900 & 1750 & 770 & 1250 & 720 & 1500 & 990 & 950 & 3000 & 510 \\
\hline $\mathrm{Ce}$ & - & 60 & 72 & 112 & 98 & 94 & 78 & - & 82 & 90 & 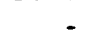 & 70 \\
\hline $\mathrm{La}$ & 30 & 54 & 68 & 98 & 82 & 72 & 66 & 30 & 74 & 78 & 70 & 64 \\
\hline $\mathrm{Nb}$ & 15 & 18 & 18 & 18 & 12 & 26 & 16 & 20 & 16 & 14 & 10 & 22 \\
\hline $\mathrm{Rb}$ & - & 148 & 54 & 102 & 126 & 80 & 94 & - & 82 & 76 & - & 166 \\
\hline $\mathrm{Sr}$ & 200 & 198 & 450 & 220 & 76 & 380 & 280 & 500 & 315 & 84 & 200 & 54 \\
\hline $\mathrm{Zr}$ & 100 & 96 & 172 & 154 & 210 & 250 & 128 & 150 & 158 & 176 & 200 & 134 \\
\hline $\bar{Y}$ & 20 & 14 & 20 & 18 & 16 & 34 & 16 & 20 & 18 & 14 & 15 & 16 \\
\hline $\mathrm{Cu}$ & 2 & $<20$ & $<20$ & $<20$ & $<20$ & $<20$ & $<20$ & 3 & $<20$ & $<20$ & 2 & $<20$ \\
\hline $\mathrm{Ni}$ & - & $<20$ & $<20$ & $<20$ & $<20$ & $<20$ & $<20$ & 1.5 & $<20$ & $<20$ & - & $<20$ \\
\hline $\mathrm{Zn}$ & - & 26 & 60 & 24 & 26 & 44 & 26 & - & 32 & 20 & - & $<20$ \\
\hline $\mathrm{Cr}$ & 5 & $<20$ & $<20$ & $<20$ & $<20$ & $<20$ & $<20$ & 5 & $<20$ & $<20$ & - & $<20$ \\
\hline
\end{tabular}

CIPW norms (weight percent)

\begin{tabular}{|c|c|c|c|c|c|c|c|c|c|c|c|c|}
\hline$Q$ & 31.17 & 31.93 & 18.23 & 31.68 & 33.28 & 25.90 & 32.94 & 24.09 & 30.26 & 30.26 & 17.78 & 33.78 \\
\hline or & 24.94 & 24.88 & 10.93 & 24.11 & 30.2 & 20.98 & 19.27 & 23.76 & 21.63 & 29.73 & 29.73 & 26.00 \\
\hline$a b$ & 26.32 & 28.26 & 30.97 & 34.19 & 29.0 & 35.12 & 35.96 & 34.86 & 34.27 & 32.24 & 42.56 & 34.86 \\
\hline an & 10.62 & 10.94 & 24.71 & 6.31 & 4.79 & 11.05 & 8.25 & 10.29 & 9.37 & 4.22 & 5.53 & 3.31 \\
\hline C & 0.29 & - & - & 0.98 & 0.69 & 0.38 & 0.83 & 0.57 & 0.61 & 1.17 & 1.45 & 0.40 \\
\hline ne & - & - & - & - & - & - & - & - & - & - & - & - \\
\hline di & - & 0.03 & 3.78 & - & - & - & - & - & - & - & - & - \\
\hline ol & - & - & - & - & - & - & - & - & - & - & - & - \\
\hline hy & 5.30 & 2.13 & 6.58 & 1.20 & 0.75 & 3.15 & 1.07 & 3.44 & 1.55 & 1.63 & 0.70 & 0.50 \\
\hline $\mathrm{mt}$ & 0.43 & 0.99 & 2.36 & 0.75 & 0.70 & 1.88 & 0.91 & 1.90 & 1.35 & 0.30 & 1.20 & 0.50 \\
\hline il & 0.61 & 0.57 & 1.65 & 0.49 & 0.38 & 0.93 & 0.49 & 0.68 & 0.57 & 0.30 & 0.42 & 0.30 \\
\hline hem & - & - & - & - & - & - & - & - & - & - & 0.28 & 0.25 \\
\hline ap & 0.19 & 0.28 & 0.76 & 0.28 & 0.19 & 0.60 & 0.28 & 0.30 & 0.37 & 0.14 & 0.23 & 0.09 \\
\hline cc & 0.11 & - & - & - & - & - & - & 0.11 & - & - & 0.11 & - \\
\hline Total & 99.98 & 100.01 & 99.97 & 99.99 & 100.00 & 99.99 & 100.00 & 100.00 & 99.98 & 99.99 & 99.99 & 99.99 \\
\hline
\end{tabular}


Table 1. Chemical analyses and norms of granitic rocks-Continued

[t, Analysis performed in the rapid rock analysis laboratory under the supervision of Leonard Shapiro; analysts: P. Elmore, S. Botts, G. Chloe, H. Smith, J. Kelsey, L. Artis and J. Glenn. *, Analysis by X-ray spectroscopy; analysts D. Vivit, M. Dyslin. Analysis of $\mathrm{FeO}, \mathrm{H}_{2} \mathrm{O}_{\text {, and }} \mathrm{CO}_{2}$ by chemical methods; analyst, N. Elsheimer. -, no data]

\begin{tabular}{|c|c|c|c|c|c|c|c|c|c|}
\hline \multirow[b]{2}{*}{$\begin{array}{l}\text { Field No. } \\
\text { Map No. }\end{array}$} & \multicolumn{2}{|c|}{$\begin{array}{c}\text { Granite of } \\
\text { Kennedy Lakes-Con. }\end{array}$} & \multirow{2}{*}{$\begin{array}{c}\begin{array}{c}\text { Granodiorite of } \\
\text { North Dome }\end{array} \\
\begin{array}{c}67 \mathrm{M} 26+ \\
\mathrm{F} 4\end{array}\end{array}$} & \multicolumn{3}{|c|}{ Pyramid pluton } & \multirow{2}{*}{$\begin{array}{c}\begin{array}{c}\text { Granodiorite of } \\
\text { Muro Blanco }\end{array} \\
\frac{68{\mathrm{M} 80^{*}}^{\mathrm{H} 2}}{}\end{array}$} & \multicolumn{2}{|c|}{$\begin{array}{l}\text { Granodiorite of } \\
\text { Lookout Peak }\end{array}$} \\
\hline & $\begin{array}{c}\text { 68M39* } \\
\text { E12 }\end{array}$ & $\begin{array}{c}\text { 68M40* } \\
\text { E11 }\end{array}$ & & $\begin{array}{c}\text { 68M35bt } \\
\text { G1(gabbro) }\end{array}$ & $\begin{array}{c}\text { 68M41t } \\
\text { G15 }\end{array}$ & $\begin{array}{c}\text { 68M42t } \\
\text { G19 (diorite) }\end{array}$ & & $\begin{array}{c}67 \mathrm{M}^{* *} \\
\mathrm{M1}\end{array}$ & $\begin{array}{c}\text { 67M1t } \\
\text { M5 }\end{array}$ \\
\hline \multicolumn{10}{|c|}{ Chemical analyses (weight percent) } \\
\hline $\mathrm{SiO}_{2}$ & 72.1 & 73.7 & 69.0 & 45.7 & 63.9 & 51.5 & 70.0 & 67.2 & 64.1 \\
\hline $\mathrm{Al}_{2}^{2} \mathrm{O}_{3}$ & 14.7 & 13.9 & 15.2 & 18.0 & 16.0 & 18.7 & 14.7 & 15.8 & 16.5 \\
\hline $\mathrm{Fe}_{2}^{2} \mathrm{O}_{3}^{3}$ & 0.77 & 0.82 & 1.80 & 4.40 & 2.80 & 4.80 & 1.18 & 1.62 & 2.50 \\
\hline $\mathrm{FeO}^{3}$ & 0.59 & 0.42 & 1.20 & 7.10 & 2.20 & 4.20 & 1.07 & 1.59 & 1.90 \\
\hline $\mathrm{MgO}$ & 0.30 & $<0.2$ & 1.20 & 6.70 & 2.30 & 4.90 & 0.70 & 1.10 & 2.00 \\
\hline $\mathrm{CaO}$ & 1.50 & 0.86 & 2.90 & 11.00 & 5.00 & 8.80 & 2.86 & 4.30 & 4.30 \\
\hline $\mathrm{Na}_{2} \mathrm{O}$ & 4.00 & 4.10 & 3.50 & 2.20 & 3.10 & 3.60 & 3.40 & 3.60 & 3.30 \\
\hline $\mathrm{K}_{2} \mathrm{O}$ & 4.58 & 4.74 & 3.60 & 0.78 & 3.00 & 0.78 & 3.94 & 2.84 & 3.60 \\
\hline $\mathrm{H}_{2}^{2} \mathrm{O}^{+}$ & 0.19 & 0.07 & 0.73 & 1.40 & 0.49 & 0.63 & 0.25 & 0.32 & 0.74 \\
\hline $\mathrm{H}_{2}^{2} \mathrm{O}^{-}$ & 0.09 & 0.11 & 0.15 & 0.11 & 0.09 & 0.14 & 0.10 & 0.07 & 0.17 \\
\hline $\mathrm{TiO}_{2}^{2}$ & 0.26 & 0.24 & 0.40 & 0.53 & 0.21 & 0.42 & 0.36 & 0.52 & 0.57 \\
\hline $\mathrm{P}_{3} \mathrm{O}_{\mathrm{s}}$ & 0.10 & 0.08 & 0.15 & 0.16 & 0.09 & 0.16 & 0.18 & 0.24 & 0.21 \\
\hline MnO & 0.06 & 0.07 & 0.07 & 1.50 & 0.66 & 1.20 & 0.05 & 0.06 & 0.09 \\
\hline LOI & 0.32 & 0.19 & - & - & $\cdot$ & - & 0.40 & 0.32 & - \\
\hline $\mathrm{CO}_{2}$ & - & - & $<.05$ & $<.05$ & 0.05 & $<.05$ & - & - & $<.05$ \\
\hline Total & 99.6 & 99.5 & 99.9 & 99.6 & 99.9 & 99.9 & 99.2 & 99.6 & 100.0 \\
\hline
\end{tabular}

Trace elements (parts per million)

\begin{tabular}{lrrrrrrrrr}
\hline $\mathrm{Ba}$ & 1600 & 990 & 1000 & 300 & 700 & 2000 & 890 & 920 & 1500 \\
$\mathrm{Ce}$ & 102 & 104 & - & - & - & - & 66 & 92 & - \\
$\mathrm{La}$ & 86 & 92 & - & - & 30 & - & 56 & 70 & 30 \\
$\mathrm{Nb}$ & 10 & 16 & 10 & - & 15 & 7 & 14 & 18 & 10 \\
$\mathrm{Rb}$ & 90 & 98 & - & - & - & - & 88 & 70 & - \\
$\mathrm{Sr}$ & 230 & 88 & 700 & 1500 & 1000 & 1500 & 325 & 520 & 1000 \\
$\mathrm{Zr}$ & 180 & 275 & 100 & 50 & 200 & 70 & 152 & 210 & 150 \\
$\mathrm{Y}$ & 10 & 14 & 15 & 50 & 30 & 20 & 14 & 20 & 20 \\
$\mathrm{Cu}$ & $<20$ & $<20$ & 10 & 50 & 30 & 100 & 20 & $<20$ & 15 \\
$\mathrm{Ni}$ & $<20$ & $<20$ & 5 & 50 & 15 & 50 & 20 & $<20$ & 10 \\
$\mathrm{Zn}$ & 24 & 30 & - & - & - & - & 28 & 38 & - \\
$\mathrm{Cr}$ & $<20$ & $<20$ & 5 & 50 & 20 & 50 & $<20$ & $<20$ & 7 \\
\hline
\end{tabular}

CIPW norms (weight percent)

\begin{tabular}{|c|c|c|c|c|c|c|c|c|c|}
\hline Q & 28.15 & 30.04 & 27.62 & - & 20.24 & 1.71 & 28.72 & 25.40 & 19.54 \\
\hline or & 27.36 & 28.25 & 21.45 & 4.67 & 17.85 & 4.67 & 23.64 & 16.96 & 21.45 \\
\hline$a b$ & 34.19 & 35.03 & 29.87 & 18.95 & 26.40 & 30.72 & 29.19 & 30.80 & 28.18 \\
\hline an & 6.89 & 3.79 & 13.24 & 37.66 & 21.04 & 32.86 & 13.26 & 18.79 & 19.76 \\
\hline C & 0.67 & 0.65 & 0.75 & - & - & - & 0.07 & 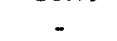 & - \\
\hline ne & - & - & - & - & - & - & - & - & - \\
\hline di & - & - & - & 13.56 & 2.43 & 7.93 & - & 0.98 & 0.06 \\
\hline ol & - & - & - & 12.86 & & - & - & - & - \\
\hline hy & 0.89 & 0.50 & 3.20 & 4.31 & 7.23 & 13.82 & 2.26 & 3.13 & 5.65 \\
\hline $\mathrm{mt}$ & 1.13 & 0.89 & 2.64 & 6.50 & 4.09 & 7.02 & 1.74 & 2.38 & 3.65 \\
\hline il & 0.49 & 0.46 & 0.76 & 1.03 & 0.40 & 0.80 & 0.70 & 1.01 & 1.10 \\
\hline hem & - & 0.22 & & - & - & - & - & 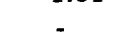 & - \\
\hline ap & 0.23 & 0.19 & 0.35 & 0.37 & 0.21 & 0.37 & 0.42 & 0.56 & 0.49 \\
\hline cc & - & - & 0.11 & 0.11 & 0.11 & 0.11 & - & $\cdot$ & 0.11 \\
\hline Total & 100.00 & 100.02 & 99.99 & 100.02 & 100.00 & 100.00 & 100.00 & 100.01 & 99.99 \\
\hline
\end{tabular}


Table 1. Chemical analyses and norms of granitic rocks-Continued

[t, Analysis performed in the rapid rock analysis laboratory under the supervision of Leonard Shapiro; analysts: P. Elmore, S. Botts, G. Chloe, H. Smith, J. Kelsey, L. Artis and J. Glenn. *, Analysis by X-ray spectroscopy; analysts D. Vivit, M. Dyslin. Analysis of FeO, $\mathrm{H}_{2} \mathrm{O}$, and $\mathrm{CO}_{2}$ by chemical methods; analyst, $\mathrm{N}$. Elsheimer. -, no data]

\begin{tabular}{|c|c|c|c|c|c|c|c|c|c|c|}
\hline \multirow[b]{2}{*}{$\begin{array}{l}\text { Field No. } \\
\text { Map No. }\end{array}$} & \multicolumn{2}{|c|}{$\begin{array}{c}\text { Granodiorite of } \\
\text { Lookout Peak-Con. }\end{array}$} & \multicolumn{5}{|c|}{ Granite of North Mountain } & \multirow{2}{*}{$\begin{array}{c}\begin{array}{c}\text { Paradise } \\
\text { pluton }\end{array} \\
\begin{array}{c}67 \mathrm{M} 86 t \\
\text { R12 }\end{array}\end{array}$} & \multicolumn{2}{|c|}{ Metavolcanic } \\
\hline & $\begin{array}{c}68 \mathrm{M} 121 * \\
\mathrm{M} 18\end{array}$ & $\begin{array}{l}\text { 68M100* } \\
M 21 \text { (diorite) }\end{array}$ & $\begin{array}{l}\text { 67M97t } \\
\text { N2 }\end{array}$ & $\begin{array}{c}\text { M794* } \\
\text { N9 }\end{array}$ & $\begin{array}{c}\text { 67M93* } \\
\text { N14 }\end{array}$ & $\begin{array}{c}68 \mathrm{M} 1 * \\
\mathrm{~N} 17\end{array}$ & $\begin{array}{l}\text { 68M2* } \\
\text { N16 }\end{array}$ & & $\begin{array}{l}\text { M183t } \\
\text { MV1 }\end{array}$ & $\begin{array}{c}68 \mathrm{M} 114 t \\
\mathrm{MV} 2\end{array}$ \\
\hline \multicolumn{11}{|c|}{ Chemical analyses (weight percent) } \\
\hline $\mathrm{SiO}_{2}$ & 69.4 & 59.4 & 73.5 & 75.8 & .76 .2 & 74.8 & 75.9 & 64.8 & 73.8 & 63.3 \\
\hline $\mathrm{Al}_{2} \mathrm{O}_{3}$ & 15.0 & 17.2 & 14.6 & 13.2 & 13.0 & 13.5 & 13.0 & 16.4 & 14.2 & 16.7 \\
\hline $\mathrm{Fe}_{2}^{2} \mathrm{O}_{3}^{3}$ & 1.35 & 2.05 & 1.10 & 0.36 & 0.44 & 0.45 & 0.23 & 2.1 & 0.60 & 2.10 \\
\hline $\mathrm{FeO}^{3}$ & 1.22 & 3.24 & 0.44 & 0.28 & 0.29 & 0.47 & 0.33 & 1.8 & 0.64 & 3.40 \\
\hline $\mathrm{MgO}$ & 0.75 & 2.30 & 0.30 & $<0.2$ & $<0.2$ & $<0.2$ & $<0.2$ & 1.8 & 0.34 & 2.20 \\
\hline $\mathrm{CaO}$ & 3.08 & 6.30 & 1.60 & 0.62 & 0.52 & 0.90 & 0.50 & 3.8 & 4.00 & 4.90 \\
\hline $\mathrm{Na}_{\mathrm{O}} \mathrm{O}$ & 3.40 & 3.80 & 3.60 & 3.60 & 3.60 & 3.70 & 3.50 & 3.4 & 0.23 & 3.00 \\
\hline $\mathrm{K}, \mathrm{O}$ & 4.08 & 2.06 & 3.80 & 5.10 & 5.04 & 4.76 & 5.30 & 4.1 & 5.00 & 2.60 \\
\hline $\mathrm{H}_{2}^{2} \mathrm{O}^{+}$ & 0.15 & 0.52 & 0.50 & 0.07 & 0.13 & 0.13 & 0.05 & 0.7 & 0.46 & 0.63 \\
\hline $\mathrm{H}_{2}^{2} \mathrm{O}^{-}$ & 0.06 & 0.08 & 0.14 & 0.05 & 0.04 & 0.05 & 0.03 & 0.1 & 0.17 & 0.09 \\
\hline $\mathrm{TiO}_{2}^{2}$ & 0.40 & 0.88 & 0.08 & 0.14 & 0.14 & 0.20 & 0.12 & 0.6 & 0.37 & 0.15 \\
\hline $\mathrm{P}_{2} \mathrm{O}_{5}$ & 0.18 & 0.44 & 0.04 & 0.06 & 0.04 & 0.08 & 0.04 & 0.2 & 0.06 & 0.12 \\
\hline MnO & 0.05 & 0.10 & 0.11 & 0.03 & 0.04 & 0.05 & 0.04 & 0.1 & 0.08 & 0.67 \\
\hline LOI & 0.18 & 0.39 & 0.17 & 0.25 & 0.23 & 0.10 & - & - & - & - \\
\hline $\mathrm{CO}_{2}$ & - & - & $<.05$ & - & - & - & - & $<.05$ & 0.05 & $<.05$ \\
\hline Total & 99.3 & 98.8 & 99.9 & 99.7 & 99.9 & 99.5 & 99.3 & 99.9 & 100.0 & 99.9 \\
\hline
\end{tabular}

Trace elements (parts per million)

\begin{tabular}{lrrrrrrrrrr}
\hline $\mathrm{Ba}$ & 700 & 840 & - & 330 & 205 & 930 & 184 & - & 2000 & 1500 \\
$\mathrm{Ce}$ & 98 & 90 & - & 90 & 78 & 80 & 66 & - & - & - \\
$\mathrm{La}$ & 82 & 74 & - & 76 & 60 & 70 & 52 & - & - & 30 \\
$\mathrm{Nb}$ & 14 & 18 & - & 18 & 24 & 34 & 16 & - & 15 & 15 \\
$\mathrm{Rb}$ & 110 & 82 & - & 162 & 172 & 220 & 186 & - & - & - \\
$\mathrm{Sr}$ & 345 & 660 & - & 48 & 30 & 170 & 30 & - & 100 & 700 \\
$\mathrm{Zr}$ & 162 & 240 & - & 126 & 88 & 164 & 84 & - & 200 & 100 \\
$\mathrm{Y}$ & 14 & 18 & - & 14 & 20 & 28 & 16 & - & 50 & 20 \\
$\mathrm{Cu}$ & $<20$ & 22 & - & $<20$ & $<20$ & $<20$ & $<20$ & - & 5 & 15 \\
$\mathrm{Ni}$ & $<20$ & $<20$ & - & $<20$ & $<20$ & $<20$ & $<20$ & - & - & 2 \\
$\mathrm{Zn}$ & 28 & 76 & - & $<20$ & $<20$ & $<20$ & $<20$ & - & - & - \\
$\mathrm{Cr}$ & $<20$ & $<20$ & - & $<20$ & $<20$ & $<20$ & $<20$ & - & 5 & 15 \\
\hline
\end{tabular}

CIPW norms (weight percent)

\begin{tabular}{lcccccccccc}
\hline Q & 26.93 & 13.6 & 34.66 & 34.10 & 34.85 & 51.76 & 34.18 & 19.02 & 44.74 & 20.27 \\
or & 24.35 & 12.47 & 22.63 & 30.32 & 29.90 & 3.01 & 31.56 & 24.47 & 29.73 & 15.48 \\
ab & 29.11 & 32.92 & 30.72 & 30.63 & 30.63 & 33.25 & 29.87 & 29.02 & 1.95 & 25.55 \\
an & 13.79 & 24.31 & 7.41 & 2.68 & 2.32 & 4.24 & 2.22 & 17.31 & 19.29 & 23.41 \\
C & - & - & 1.88 & 0.79 & 0.78 & 5.66 & 0.71 & 0.07 & 1.40 & 0.46 \\
ne & - & - & - & - & - & - & - & - & - & - \\
di & 0.37 & 3.81 & - & - & - & - & - & - & - & - \\
ol & - & - & - & - & - & - & - & - & - & - \\
hy & 2.28 & 7.05 & 0.75 & 0.54 & 0.51 & 0.80 & 0.79 & 5.32 & 1.06 & 11.09 \\
mt & 1.97 & 3.04 & 1.55 & 0.52 & 0.64 & 0.68 & 0.33 & 3.07 & 0.87 & 3.07 \\
il & 0.76 & 1.71 & 0.15 & 0.27 & 0.27 & 0.40 & 0.23 & 1.12 & 0.70 & 0.28 \\
hem & -.42 & - & 0.04 & - & - & - & - & - & - & - \\
ap & 0.42 & 1.04 & 0.09 & 0.14 & 0.09 & 0.19 & 0.09 & 0.49 & 0.14 & 0.28 \\
cc & - & - & $\mathbf{0 . 1 1}$ & - & - & - & - & 0.11 & 0.11 & 0.11 \\
\multicolumn{1}{r}{ Total } & 99.98 & 99.99 & 99.99 & $\mathbf{9 9 . 9 9}$ & $\mathbf{9 9 . 9 9}$ & $\mathbf{9 9 . 9 9}$ & $\mathbf{9 9 . 9 8}$ & 100.00 & 99.99 & 100.00 \\
\hline
\end{tabular}


Table 2. Modal analyses of granitic rocks

[Analyst, Oleg Polovtzoff. S.D., standard deviation. - , no data]

\begin{tabular}{llllllll}
\hline $\begin{array}{l}\text { Map } \\
\text { No. }\end{array}$ & Field & Quartz & K-feldspar & Plagioclase & $\begin{array}{c}\text { Mafic } \\
\text { minerals }\end{array}$ & $\begin{array}{c}\text { Specific } \\
\text { gravity }\end{array}$ & $\begin{array}{c}\text { Magnetic } \\
\text { susceptibility }\end{array}$ \\
\hline
\end{tabular}

Granite of Tehipite Dome

\begin{tabular}{|c|c|c|c|c|c|c|c|}
\hline A1 & M163 & 27.4 & 14.4 & 47.2 & 11.1 & - & - \\
\hline A3 & $67 \mathrm{M} 41$ & 21.0 & 24.4 & 42.9 & 11.7 & 2.65 & 1480 \\
\hline A4 & 67M50 & 26.0 & 26.8 & 37.4 & 9.8 & 2.66 & 1520 \\
\hline A5 & $67 \mathrm{M} 25$ & 27.9 & 31.2 & 33.7 & 7.2 & 2.62 & 1580 \\
\hline A6 & $67 \mathrm{M} 24$ & 26.2 & 29.4 & 36.2 & 8.2 & 2.64 & 2000 \\
\hline A7 & $67 \mathrm{M} 47$ & 20.8 & 20.3 & 43.5 & 15.4 & 2.68 & 2200 \\
\hline A8 & $68 \mathrm{M} 50$ & 36.2 & 32.3 & 21.6 & 9.9 & - & 1280 \\
\hline A9 & $68 \mathrm{M} 51$ & 45.2 & 11.5 & 16.4 & 26.9 & - & 2600 \\
\hline \multirow{2}{*}{\multicolumn{2}{|c|}{$\begin{array}{l}\text { Average } \\
\text { S.D. }\end{array}$}} & 28.8 & 23.8 & 34.9 & 12.5 & 2.65 & 1768 \\
\hline & & 8.2 & 7.2 & 10.1 & 5.9 & 0.02 & 423 \\
\hline
\end{tabular}

Granite of Alpine Creek

\begin{tabular}{|c|c|c|c|c|c|c|c|}
\hline B1 & M736 & 32.6 & 29.2 & 35.9 & 2.3 & - & - \\
\hline B2 & M735 & 43.3 & 24.6 & 26.2 & 5.9 & - & 1680 \\
\hline B3 & M742 & 27.5 & 36.0 & 33.3 & 3.2 & - & 1560 \\
\hline B4 & M773 & 30.6 & 32.7 & 36.1 & 0.6 & - & 1200 \\
\hline \multirow{2}{*}{\multicolumn{2}{|c|}{$\begin{array}{l}\text { Average-1- }-1 \\
\text { S.D. }\end{array}$}} & 33.5 & 30.6 & 32.9 & 3.0 & - & 1480 \\
\hline & & 5.9 & 4.2 & 4.0 & 1.9 & - & 204 \\
\hline
\end{tabular}

\begin{tabular}{|c|c|c|c|c|c|c|c|}
\hline C1 & M512 & 24.3 & 19.0 & 52.1 & 4.6 & 2.63 & 1900 \\
\hline $\mathrm{C} 2$ & M511 & 26.2 & 41.1 & 31.1 & 1.6 & 2.61 & 640 \\
\hline C3 & M94 & 30.0 & 20.5 & 47.0 & 2.5 & 2.62 & 980 \\
\hline $\mathrm{C} 4$ & M536 & 28.9 & 22.8 & 44.8 & 3.5 & 2.75 & 1500 \\
\hline C5 & M112 & 27.5 & 21.2 & 46.7 & 4.6 & 2.65 & 3200 \\
\hline C6 & M116 & 22.7 & 22.7 & 50.1 & 4.5 & 2.67 & 4200 \\
\hline C7 & $64 \mathrm{M} 8$ & 35.8 & 25.6 & 35.4 & 3.2 & 2.62 & 1860 \\
\hline $\mathrm{C} 8$ & M110 & 27.6 & 23.6 & 44.3 & 4.7 & 2.66 & 1920 \\
\hline $\mathrm{C} 9$ & $64 \mathrm{M} 20$ & 42.7 & 25.9 & 18.8 & 12.6 & - & 3400 \\
\hline $\mathrm{C} 10$ & M111 & 24.0 & 25.5 & 44.5 & 6.0 & 2.66 & - \\
\hline $\mathrm{C} 11$ & M528 & 37.4 & 2.7 & 51.4 & 8.5 & 2.69 & 800 \\
\hline $\mathrm{C} 12$ & $64 \mathrm{M} 6$ & 28.3 & 28.8 & 38.5 & 4.4 & - & 2400 \\
\hline $\mathrm{C} 13$ & 68M31 & 35.6 & 33.4 & 30.0 & 1.0 & 2.58 & 900 \\
\hline $\mathrm{C} 14$ & M534 & 19.0 & 21.6 & 49.4 & 10.0 & 2.67 & 2600 \\
\hline C15 & M533 & 26.5 & 15.8 & 41.4 & 16.3 & 2.71 & 1720 \\
\hline $\mathrm{C} 16$ & M532 & 24.0 & 19.1 & 51.6 & 5.3 & 2.64 & 1300 \\
\hline $\mathrm{C} 17$ & M526 & 33.0 & 23.8 & 39.8 & 3.4 & 2.64 & 1820 \\
\hline C18 & $64 \mathrm{M} 37$ & 45.5 & 26.4 & 23.1 & 5.0 & - & 1260 \\
\hline C19 & M74 & • 35.4 & 23.4 & 37.6 & 3.6 & 2.65 & 3000 \\
\hline $\mathrm{C} 20$ & M531 & 30.4 & 17.9 & 45.2 & 6.5 & 2.66 & - \\
\hline C21 & M530 & 20.9 & 16.5 & 53.8 & 8.8 & 2.66 & 2200 \\
\hline $\mathrm{C} 22$ & $64 \mathrm{M} 3$ & 22.3 & 28.8 & 41.4 & 7.5 & + & 2800 \\
\hline $\mathrm{C} 23$ & $64 \mathrm{M} 9$ & 26.4 & 26.4 & 42.3 & 4.9 & - & 3000 \\
\hline C24 & $64 \mathrm{M} 4$ & 26.5 & 23.7 & 43.4 & 6.4 & 2.64 & 3000 \\
\hline $\mathrm{C} 25$ & $64 \mathrm{M} 10$ & 39.8 & 23.5 & 27.2 & 9.5 & - & 2200 \\
\hline \multirow{2}{*}{\multicolumn{2}{|c|}{ 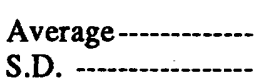 }} & 29.6 & 23.1 & 41.2 & 6.0 & 2.65 & 2113 \\
\hline & & 6.8 & 6.7 & 9.1 & 3.4 & 0.04 & 913 \\
\hline
\end{tabular}

Cartridge Pass Pluton

\begin{tabular}{llllllll}
\hline D1 & M60 & 24.9 & 21.7 & 47.5 & 5.9 & 2.67 & - \\
D2 & M58 & 24.3 & 21.6 & 45.4 & 8.7 & 2.68 & -
\end{tabular}


Table 2. Modal analyses of granitic rocks-Continued

[Analyst, Oleg Polovtzoff. S.D., standard deviation. - , no data]

\begin{tabular}{lccccccc}
\hline $\begin{array}{l}\text { Map } \\
\text { No. }\end{array}$ & $\begin{array}{c}\text { Field } \\
\text { No. }\end{array}$ & Quartz & K-feldspar & Plagioclase & $\begin{array}{c}\text { Mafic } \\
\text { minerals }\end{array}$ & $\begin{array}{c}\text { Specific } \\
\text { gravity }\end{array}$ & $\begin{array}{c}\text { Magnetic } \\
\text { susceptibility }\end{array}$ \\
\hline \multicolumn{7}{c}{ Cartridge Pass Pluton-Continued } \\
\hline D3 & M57 & 27.2 & 19.2 & 46.9 & 6.7 & 2.67 & - \\
D4 & M63 & 22.6 & 14.7 & 53.9 & 8.8 & 2.67 & - \\
D5 & M61 & 28.4 & $\underline{19.4}$ & $\underline{45.0}$ & $\frac{7.2}{7.9}$ & $\frac{2.66}{2.67}$ & - \\
Average & & $\underline{25.6}$ & $\underline{19.3}$ & $\overline{47.2}$ & 7.9 & - \\
S.D. & 1.9 & 2.3 & 3.2 & 1.4 & 0.01 & - \\
\hline
\end{tabular}

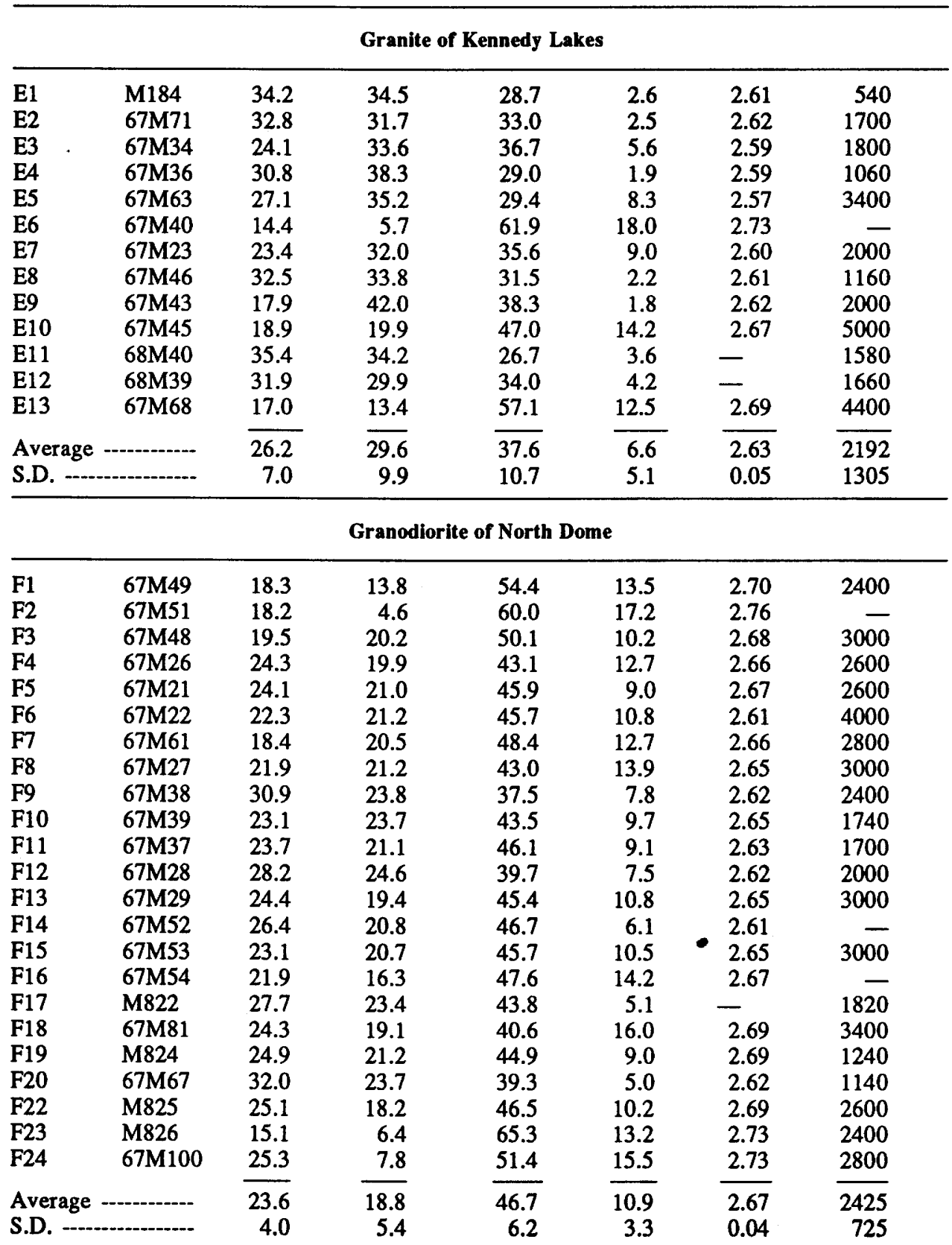


Table 2. Modal analyses of granitic rocks-Continued

[Analyst, Oleg Polovtzoff. S.D., standard deviation. -, no data]

\begin{tabular}{lccccccc}
\hline $\begin{array}{l}\text { Map } \\
\text { No. }\end{array}$ & $\begin{array}{c}\text { Field } \\
\text { No. }\end{array}$ & Quartz & K-feldspar & Plagioclase & $\begin{array}{c}\text { Mafic } \\
\text { minerals }\end{array}$ & $\begin{array}{l}\text { Specific } \\
\text { gravity }\end{array}$ & $\begin{array}{c}\text { Magnetic } \\
\text { susceptibility }\end{array}$ \\
\hline
\end{tabular}

\begin{tabular}{|c|c|c|c|c|c|c|c|}
\hline \multicolumn{8}{|c|}{ Pyramid pluton } \\
\hline G1 & $68 \mathrm{M} 35 \mathrm{~b}$ & - & - & - & - & 一 & 14800 \\
\hline G2 & 68M56 & 12.3 & 3.3 & 66.7 & 17.7 & 2.78 & 7000 \\
\hline G3 & 68M57 & 11.7 & 8.7 & 58.2 & 21.4 & 2.75 & 5600 \\
\hline G4 & $64 \mathrm{M} 11$ & 18.1 & 8.0 & 57.6 & 16.3 & 2.77 & 4600 \\
\hline G5 & $64 \mathrm{M} 15$ & 19.8 & 13.0 & 51.1 & 16.1 & 2.74 & 4400 \\
\hline G6 & $64 \mathrm{M} 14$ & 16.0 & 18.2 & 52.3 & 13.5 & 2.72 & 5200 \\
\hline G7 & $68 \mathrm{M} 79$ & 18.3 & 13.7 & 49.3 & 18.7 & 2.72 & 4600 \\
\hline G8 & $64 \mathrm{M} 16$ & 20.2 & 9.6 & 50.6 & 19.5 & 2.73 & 5400 \\
\hline G9 & $68 \mathrm{M} 76$ & 19.2 & 13.7 & 48.3 & 18.8 & 2.72 & 5000 \\
\hline G10 & $68 \mathrm{M} 75$ & 20.8 & 13.8 & 47.9 & 17.5 & 2.73 & 5000 \\
\hline G11 & $68 \mathrm{M} 77$ & 24.9 & 20.3 & 42.7 & 12.1 & 2.69 & 3800 \\
\hline G12 & $68 \mathrm{M} 9$ & 22.7 & 16.7 & 44.5 & 16.1 & 2.70 & 6200 \\
\hline G13 & $68 \mathrm{M} 10$ & 22.5 & 18.8 & 43.8 & 14.9 & 2.68 & 5200 \\
\hline G14 & $68 \mathrm{M} 74$ & 20.6 & 25.0 & 41.3 & 13.1 & 2.71 & 4800 \\
\hline G15 & $68 \mathrm{M} 41$ & 22.6 & 13.0 & 47.6 & 16.8 & 2.73 & 5000 \\
\hline G16 & $68 \mathrm{M} 60$ & 19.2 & 12.7 & 54.8 & 13.3 & 2.70 & 3200 \\
\hline G17 & 68M59 & 18.3 & 8.9 & 59.2 & 13.6 & 2.74 & 3800 \\
\hline G18 & $68 \mathrm{M} 58$ & 19.5 & 16.8 & 46.4 & 17.3 & 2.73 & 4600 \\
\hline G19 & $68 \mathrm{M} 42$ & - & - & - & - & - & 13000 \\
\hline G20 & $64 \mathrm{M} 33$ & 24.5 & 22.1 & 42.4 & 11.0 & 2.69 & 4000 \\
\hline \multirow{2}{*}{\multicolumn{2}{|c|}{$\begin{array}{l}\text { Average }-. . \\
\text { S.D. }\end{array}$}} & 18.6 & 13.0 & 51.6 & 16.8 & 2.73 & 5855 \\
\hline & & 4.6 & 6.3 & 7.5 & 3.9 & 0.04 & 2800 \\
\hline
\end{tabular}

\begin{tabular}{|c|c|c|c|c|c|c|c|}
\hline \multicolumn{8}{|c|}{ Granodiorite of Muro Blanco } \\
\hline $\begin{array}{l}\mathrm{H} 1 \\
\mathrm{H} 2\end{array}$ & $\begin{array}{l}\text { B794 } \\
68 \mathrm{M} 80\end{array}$ & $\begin{array}{l}22.7 \\
41.6\end{array}$ & $\begin{array}{l}23.8 \\
25.4\end{array}$ & $\begin{array}{l}39.6 \\
25.0\end{array}$ & $\begin{array}{r}13.9 \\
8.0\end{array}$ & - & $\begin{array}{l}2200 \\
4000\end{array}$ \\
\hline $\begin{array}{l}\text { Aver } \\
\text { S.D. }\end{array}$ & - & $\begin{array}{r}32.2 \\
9.5\end{array}$ & $\begin{array}{l}22.4 \\
13.3\end{array}$ & $\begin{array}{l}25.9 \\
15.4\end{array}$ & $\begin{array}{l}6.7 \\
4.0\end{array}$ & - & $\begin{array}{r}2000 \\
900\end{array}$ \\
\hline
\end{tabular}

\begin{tabular}{|c|c|c|c|c|c|c|c|}
\hline \multicolumn{8}{|c|}{ Arrow pluton } \\
\hline I1 & B792 & 25.4 & 22.0 & 46.2 & 6.4 & - & 3000 \\
\hline $\mathrm{I} 2$ & B785 & 27.0 & 14.8 & 44.5 & 13.6 & 2.69 & - \\
\hline I3 & B787 & 27.9 & 16.6 & 42.8 & 12.7 & 2.69 & 一 \\
\hline Av & - & 26.8 & 17.8 & 44.5 & 10.9 & 2.69 & 3000 \\
\hline S.I & ------ & 1.0 & 3.1 & 1.4 & 3.2 & - & - \\
\hline \multicolumn{8}{|c|}{ Granite of Grand Dike } \\
\hline J1 & $68 \mathrm{M} 117$ & 32.1 & 40.8 & 25.9 & 1.2 & 2.62 & 7200 \\
\hline
\end{tabular}

\begin{tabular}{|c|c|c|c|c|c|c|c|}
\hline \multicolumn{8}{|c|}{ Granodiorite of Lightning Creek } \\
\hline K1 & B860 & 20.7 & 10.4 & 45.6 & 23.3 & - & - \\
\hline $\mathbf{K} 2$ & M809 & 23.4 & 12.3 & 45.5 & 18.8 & - & 3200 \\
\hline $\mathrm{K} 3$ & M807 & 19.0 & 7.3 & 55.7 & 18.0 & 2.79 & 4600 \\
\hline K4 & M806 & 17.9 & 10.2 & 52.1 & 19.8 & 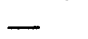 & 4200 \\
\hline K5 & M805 & 17.1 & 9.8 & 53.5 & 19.6 & - & 5000 \\
\hline K6 & M193 & 23.4 & 14.5 & 46.7 & 15.4 & 2.71 & 1100 \\
\hline K7 & $2 / 37$ & 16.4 & 6.4 & 54.0 & 23.2 & 2.78 & 6000 \\
\hline K8 & $68 \mathrm{M} 123$ & 21.3 & 12.5 & 49.2 & 17.0 & 2.72 & 6000 \\
\hline \multirow{2}{*}{\multicolumn{2}{|c|}{$\begin{array}{l}\text { Average - }-1 .-1 \\
\text { S.D. }\end{array}$}} & 19.9 & 10.4 & 50.3 & 19.4 & 2.75 & 4300 \\
\hline & & 2.5 & 2.5 & 3.8 & 2.6 & 0.04 & 1596 \\
\hline
\end{tabular}


Table 2. Modal analyses of granitic rocks-Continued

[Analyst, Oleg Polovtzoff. S.D., standard deviation. -, no data]

\begin{tabular}{|c|c|c|c|c|c|c|c|}
\hline $\begin{array}{l}\text { Map } \\
\text { No. }\end{array}$ & $\begin{array}{l}\text { Field } \\
\text { No. }\end{array}$ & Quartz & K-feldspar & Plagioclase & $\begin{array}{c}\text { Mafic } \\
\text { minerals }\end{array}$ & $\begin{array}{c}\text { Specific } \\
\text { gravity }\end{array}$ & $\begin{array}{c}\text { Magnetic } \\
\text { susceptibility }\end{array}$ \\
\hline \multicolumn{8}{|c|}{ Granodiorite of Mitchell Peak } \\
\hline $\begin{array}{l}\mathrm{L} 1 \\
\mathrm{~L} 2 \\
\mathrm{~L} 3\end{array}$ & $\begin{array}{l}\text { M167 } \\
2 / 36 \\
68 \mathrm{M} 98\end{array}$ & $\begin{array}{l}16.9 \\
14.3 \\
21.4\end{array}$ & $\begin{array}{l}17.4 \\
10.7 \\
15.0\end{array}$ & $\begin{array}{l}47.2 \\
55.0 \\
51.7\end{array}$ & $\begin{array}{l}18.5 \\
20.0 \\
11.9\end{array}$ & $\begin{array}{l}2.74 \\
2.75 \\
2.71\end{array}$ & $\begin{array}{l}4000 \\
3400 \\
5000\end{array}$ \\
\hline $\begin{array}{l}\text { Average } \\
\text { S.D. }\end{array}$ & - & $\begin{array}{r}17.5 \\
2.9\end{array}$ & $\begin{array}{r}14.4 \\
2.8\end{array}$ & $\begin{array}{r}51.3 \\
3.2\end{array}$ & $\begin{array}{r}16.8 \\
3.5\end{array}$ & $\begin{array}{l}2.73 \\
0.02\end{array}$ & $\begin{array}{r}4133 \\
660\end{array}$ \\
\hline \multicolumn{8}{|c|}{ Granodiorite of Lookout Peak } \\
\hline $\begin{array}{l}\text { M1 } \\
\text { M2 } \\
\text { M4 } \\
\text { M5 } \\
\text { M6 } \\
\text { M7 } \\
\text { M8 } \\
\text { M10 } \\
\text { M11 } \\
\text { M12 } \\
\text { M13 } \\
\text { M14 } \\
\text { M15 } \\
\text { M16 } \\
\text { M17 } \\
\text { M18 } \\
\text { M19 } \\
\text { M20 } \\
\text { M21 } \\
\text { M22 }\end{array}$ & $\begin{array}{l}67 \mathrm{M} 76 \\
\text { M186 } \\
68 \mathrm{M} 92 \\
68 \mathrm{M} 88 \\
67 \mathrm{M} 1 \\
67 \mathrm{M} 10 \\
68 \mathrm{M} 102 \\
\mathrm{M} 195 \\
67 \mathrm{M} 83 \\
\mathrm{M} 168 \\
67 \mathrm{M} 8 \\
67 \mathrm{M} 9 \\
67 \mathrm{M} 3 \\
\mathrm{M} 170 \\
67 \mathrm{M} 4 \\
67 \mathrm{M} 5 \\
68 \mathrm{M} 121 \\
67 \mathrm{M} 91 \\
68 \mathrm{M} 97 \\
68 \mathrm{M} 100 \\
68 \mathrm{M} 89\end{array}$ & $\begin{array}{l}22.1 \\
27.2 \\
25.7 \\
18.2 \\
20.8 \\
21.9 \\
17.4 \\
19.5 \\
22.8 \\
27.5 \\
22.0 \\
20.9 \\
21.8 \\
17.3 \\
20.6 \\
16.4 \\
36.1 \\
16.0 \\
16.7 \\
55.2 \\
29.6\end{array}$ & $\begin{array}{r}16.7 \\
2.0 \\
28.5 \\
12.2 \\
12.3 \\
7.1 \\
7.8 \\
14.6 \\
16.5 \\
26.8 \\
7.6 \\
10.1 \\
9.8 \\
13.3 \\
7.2 \\
19.2 \\
33.4 \\
9.7 \\
8.6 \\
8.0 \\
5.5\end{array}$ & $\begin{array}{l}45.0 \\
50.0 \\
38.9 \\
52.9 \\
46.7 \\
52.7 \\
51.9 \\
48.7 \\
42.5 \\
38.3 \\
52.8 \\
49.0 \\
52.2 \\
52.0 \\
53.7 \\
56.7 \\
21.2 \\
56.1 \\
57.7 \\
11.0 \\
44.6\end{array}$ & $\begin{array}{r}16.2 \\
20.8 \\
6.9 \\
16.7 \\
20.2 \\
18.3 \\
22.9 \\
17.2 \\
18.2 \\
7.4 \\
17.6 \\
20.0 \\
16.2 \\
17.4 \\
18.5 \\
7.7 \\
9.3 \\
18.2 \\
17.0 \\
25.8 \\
20.3\end{array}$ & $\begin{array}{l}2.72 \\
2.73 \\
2.62 \\
2.69 \\
2.69 \\
2.70 \\
2.72 \\
2.75 \\
2.70 \\
2.64 \\
2.72 \\
2.73 \\
2.74 \\
2.73 \\
2.63 \\
\\
2.77 \\
2.70 \\
-\end{array}$ & $\begin{array}{l}3800 \\
3000 \\
3400 \\
5000 \\
4200 \\
7000 \\
4800 \\
4800 \\
5200 \\
3800 \\
6000 \\
8000 \\
5600 \\
5200 \\
4000 \\
1900 \\
2200 \\
4800 \\
5600 \\
5200 \\
4600\end{array}$ \\
\hline $\begin{array}{l}\text { Average } \\
\text { S.D. }\end{array}$ & --.--...... & $\begin{array}{r}23.1 \\
9.2\end{array}$ & $\begin{array}{r}13.2 \\
7.8\end{array}$ & $\begin{array}{l}46.4 \\
11.2\end{array}$ & $\begin{array}{r}16.8 \\
4.9\end{array}$ & $\begin{array}{l}2.70 \\
0.04\end{array}$ & $\begin{array}{l}\overline{4605} \\
1405\end{array}$ \\
\hline \multicolumn{8}{|c|}{ Granodiorite of North Mountain } \\
\hline $\begin{array}{l}\text { N1 } \\
\text { N2 } \\
\text { N3 } \\
\text { N5 } \\
\text { N6 } \\
\text { N7 } \\
\text { N8 } \\
\text { N9 } \\
\text { N10 } \\
\text { N11 } \\
\text { N12 } \\
\text { N13 } \\
\text { N14 } \\
\text { N15 } \\
\text { N16 } \\
\text { N17 }\end{array}$ & $\begin{array}{l}\text { 67M96 } \\
67 \mathrm{M} 97 \\
\text { 67M99 } \\
\text { 67M98 } \\
\text { M83 } \\
\text { M82 } \\
\text { M81 } \\
\text { M175 } \\
\text { M794 } \\
\text { M176 } \\
\text { 67M90 } \\
\text { 67M95 } \\
\text { 67M94 } \\
\text { 67M93 } \\
\text { 67M92 } \\
\text { 68M2 } \\
\text { 68M1 }\end{array}$ & $\begin{array}{r}23.2 \\
28.0 \\
5.0 \\
4.6 \\
32.7 \\
33.8 \\
33.2 \\
27.9 \\
30.3 \\
20.4 \\
22.5 \\
5.0 \\
1.9 \\
3.4 \\
9.0 \\
20.4 \\
30.4\end{array}$ & $\begin{array}{r}27.3 \\
24.2 \\
30.3 \\
33.3 \\
5.8 \\
44.4 \\
44.9 \\
38.9 \\
39.3 \\
57.6 \\
22.8 \\
47.5 \\
40.9 \\
55.1 \\
35.1 \\
52.3 \\
39.1\end{array}$ & $\begin{array}{l}39.9 \\
43.9 \\
59.3 \\
60.3 \\
58.1 \\
20.1 \\
20.9 \\
30.4 \\
28.5 \\
21.4 \\
48.5 \\
45.1 \\
56.4 \\
40.5 \\
50.0 \\
25.4 \\
28.6\end{array}$ & $\begin{array}{l}9.6 \\
3.9 \\
5.4 \\
1.8 \\
3.4 \\
1.7 \\
1.0 \\
2.8 \\
1.9 \\
0.6 \\
6.1 \\
2.4 \\
0.8 \\
1.0 \\
5.9 \\
1.9 \\
1.9\end{array}$ & $\begin{array}{l}2.66 \\
2.61 \\
2.64 \\
2.62 \\
2.68 \\
2.60 \\
2.60 \\
2.61 \\
- \\
2.60 \\
2.65 \\
2.62 \\
2.60 \\
2.61 \\
2.66 \\
-\end{array}$ & $\begin{array}{r}2800 \\
700 \\
1600 \\
-\overline{1740} \\
640 \\
920 \\
980 \\
960 \\
880 \\
2600 \\
820 \\
44 \\
1020 \\
1400 \\
1160 \\
1420\end{array}$ \\
\hline $\begin{array}{l}\text { Average } \\
\text { S.D. }\end{array}$ & - & $\begin{array}{l}19.5 \\
11.6\end{array}$ & $\begin{array}{l}\overline{38.8} \\
13.3\end{array}$ & $\begin{array}{l}\overline{39.8} \\
13.9\end{array}$ & $\begin{array}{l}3.1 \\
2.4\end{array}$ & $\begin{array}{l}\overline{2.63} \\
0.03\end{array}$ & $\begin{array}{r}1230 \\
681\end{array}$ \\
\hline
\end{tabular}


Table 2. Modal analyses of granitic rocks - Continued

[Analyst, Oleg Polovtzoff. S.D., standard deviation. -, no data]

\begin{tabular}{|c|c|c|c|c|c|c|c|}
\hline $\begin{array}{l}\text { Map } \\
\text { No. }\end{array}$ & $\begin{array}{l}\text { Field } \\
\text { No. }\end{array}$ & Quartz & K-feldspar & Plagioclase & $\begin{array}{c}\text { Mafic } \\
\text { minerals }\end{array}$ & $\begin{array}{l}\text { Specific } \\
\text { gravity }\end{array}$ & $\begin{array}{c}\text { Magnetic } \\
\text { susceptibility }\end{array}$ \\
\hline \multicolumn{8}{|c|}{ Granodiorite of Sugarloaf } \\
\hline $\begin{array}{l}\text { P1 } \\
\text { P2 } \\
\text { P3 } \\
\text { P4 }\end{array}$ & $\begin{array}{l}2 / 33 \\
68 \mathrm{M} 95 \\
68 \mathrm{M} 201 \\
68 \mathrm{M} 19\end{array}$ & $\begin{array}{l}20.0 \\
21.7 \\
22.1 \\
19.7\end{array}$ & $\begin{array}{l}13.1 \\
17.8 \\
21.9 \\
18.4\end{array}$ & $\begin{array}{l}49.1 \\
44.4 \\
46.2 \\
44.2\end{array}$ & $\begin{array}{r}17.8 \\
16.1 \\
9.8 \\
17.7\end{array}$ & $\begin{array}{l}2.74 \\
2.70 \\
2.69 \\
2.71\end{array}$ & $\begin{array}{l}5400 \\
4400 \\
3800 \\
4800\end{array}$ \\
\hline $\begin{array}{l}\text { Averag } \\
\text { S.D. - }\end{array}$ & - & $\begin{array}{r}20.9 \\
1.0\end{array}$ & $\begin{array}{r}17.8 \\
3.2\end{array}$ & $\begin{array}{r}46.0 \\
2.0\end{array}$ & $\begin{array}{r}15.4 \\
3.3\end{array}$ & $\begin{array}{l}2.71 \\
0.02\end{array}$ & $\begin{array}{r}4600 \\
583\end{array}$ \\
\hline \multicolumn{8}{|c|}{ Paradise pluton } \\
\hline $\begin{array}{l}\text { R1 } \\
\text { R2 } \\
\text { R3 } \\
\text { R4 } \\
\text { R5 } \\
\text { R6 } \\
\text { R7 } \\
\text { R8 } \\
\text { R9 } \\
\text { R10 } \\
\text { R11 } \\
\text { R12 } \\
\text { R13 } \\
\text { R14 } \\
\text { R15 } \\
\text { R16 } \\
\text { R17 } \\
\text { R18 } \\
\text { R19 } \\
\text { R20 } \\
\text { R21 } \\
\text { R22 } \\
\text { R23 }\end{array}$ & $\begin{array}{l}\text { M70 } \\
\text { M69 } \\
68 \mathrm{M} 6 \\
\text { B-798 } \\
\text { B-799 } \\
\text { B-950 } \\
67 \mathrm{M} 88 \\
\text { M68 } \\
\text { M67 } \\
67 \mathrm{M} 7 \\
67 \mathrm{M} 87 \\
67 \mathrm{M} 86 \\
67 \mathrm{M} 85 \\
67 \mathrm{M} 89 \\
\text { 2/31 } \\
67 \mathrm{M} 20 \\
67 \mathrm{M} 19 \\
67 \mathrm{M} 11 \\
67 \mathrm{M} 18 \\
67 \mathrm{M} 17 \\
67 \mathrm{M} 14 \\
67 \mathrm{M} 15 \\
67 \mathrm{M} 12\end{array}$ & $\begin{array}{l}24.3 \\
24.2 \\
22.0 \\
19.3 \\
23.8 \\
19.3 \\
19.9 \\
27.5 \\
24.1 \\
28.7 \\
23.2 \\
23.4 \\
19.0 \\
23.5 \\
29.0 \\
25.2 \\
23.6 \\
24.6 \\
18.9 \\
23.1 \\
19.2 \\
21.3 \\
26.2\end{array}$ & $\begin{array}{l}16.1 \\
15.8 \\
22.6 \\
16.8 \\
19.3 \\
18.7 \\
22.1 \\
16.0 \\
15.8 \\
13.6 \\
14.7 \\
12.1 \\
10.8 \\
15.7 \\
23.0 \\
19.0 \\
20.8 \\
13.9 \\
27.5 \\
20.9 \\
23.2 \\
15.4 \\
18.6\end{array}$ & $\begin{array}{l}47.3 \\
51.4 \\
48.0 \\
46.5 \\
45.4 \\
47.3 \\
46.0 \\
48.9 \\
47.7 \\
41.2 \\
48.2 \\
50.2 \\
54.9 \\
47.8 \\
41.6 \\
44.3 \\
43.8 \\
48.2 \\
41.2 \\
45.0 \\
44.9 \\
50.5 \\
43.6\end{array}$ & $\begin{array}{r}12.3 \\
8.6 \\
7.4 \\
17.4 \\
11.4 \\
14.7 \\
12.0 \\
7.6 \\
12.4 \\
16.5 \\
13.9 \\
14.3 \\
15.3 \\
13.0 \\
6.4 \\
11.5 \\
11.8 \\
13.3 \\
12.4 \\
11.0 \\
12.7 \\
12.8 \\
11.5\end{array}$ & $\begin{array}{l}2.72 \\
2.70 \\
2.68 \\
2.68 \\
2.70 \\
2.69 \\
2.62 \\
2.67 \\
2.70 \\
2.67 \\
2.71 \\
2.69 \\
2.71 \\
2.67 \\
2.66 \\
2.68 \\
2.68 \\
2.69 \\
2.68 \\
2.67 \\
2.67 \\
2.69 \\
2.66\end{array}$ & $\begin{array}{r}3800 \\
3800 \\
4000 \\
- \\
- \\
- \\
4000 \\
2400 \\
4000 \\
4400 \\
4200 \\
3200 \\
4400 \\
5000 \\
3000 \\
5200 \\
3800 \\
4000 \\
4200 \\
2600 \\
3400 \\
4000 \\
3000\end{array}$ \\
\hline \multicolumn{2}{|c|}{$\begin{array}{l}\text { Average- } \\
\text { S.D. }\end{array}$} & $\begin{array}{r}23.2 \\
2.9\end{array}$ & $\begin{array}{r}17.9 \\
4.0\end{array}$ & $\begin{array}{r}46.7 \\
3.3\end{array}$ & $\begin{array}{r}12.2 \\
2.7\end{array}$ & $\begin{array}{l}2.68 \\
0.02\end{array}$ & $\begin{array}{r}3820 \\
701\end{array}$ \\
\hline
\end{tabular}


Table 3. Chemical analyses and norms of Cenozoic volcanic rocks

\begin{tabular}{|c|c|c|c|c|c|c|c|c|c|}
\hline $\begin{array}{l}\text { Field No. } \\
\text { Map No. }\end{array}$ & $\begin{array}{l}\text { M117 } \\
\text { CC-2 }\end{array}$ & $\begin{array}{c}M-778 \\
C C-3\end{array}$ & $\begin{array}{l}69 \mathrm{M} 9 \\
\mathrm{CC}-4\end{array}$ & $\begin{array}{c}M-113 \\
C C-5\end{array}$ & $\begin{array}{c}68 \mathrm{M} 32 \\
\mathrm{CC}-7\end{array}$ & $\begin{array}{c}67 \mathrm{M74} \\
\mathrm{CC}-8\end{array}$ & $\begin{array}{l}67 M 78 \\
C C-10\end{array}$ & $\begin{array}{l}67 M 66 \\
C C-11\end{array}$ & $\begin{array}{l}67 M 84 \\
C C-12\end{array}$ \\
\hline \multicolumn{10}{|c|}{ Chemical analyses (weight percent) } \\
\hline $\mathrm{SiO}_{2}$ & 63.0 & 47.9 & 48.92 & 63.3 & 53.7 & 47.9 & 48.13 & 57.17 & 49.5 \\
\hline $\mathrm{Al}_{2} \mathrm{O}_{3}$ & 17.5 & 13.6 & 13.39 & 17.3 & 13.9 & 11.8 & 13.78 & 14.94 & 13.3 \\
\hline $\mathrm{Fe}_{2}^{2} \mathrm{O}_{3}^{3}$ & 2.1 & 4.5 & 4.51 & 2.6 & 5.7 & 6.2 & 4.27 & 2.79 & 5.7 \\
\hline $\mathrm{FeO}^{2}$ & 1.7 & 4.6 & 3.52 & 1.2 & 1.4 & 2.8 & 4.00 & 2.83 & 3.4 \\
\hline $\mathrm{MgO}$ & 1.6 & 11.7 & 7.44 & 2.1 & 6.4 & 11.1 & 11.61 & 5.94 & 10.2 \\
\hline $\mathrm{CaO}$ & 3.6 & 9.7 & 9.12 & 4.2 & 6.8 & 8.4 & 9.36 & 5.73 & 9.4 \\
\hline $\mathrm{Na}_{2} \mathrm{O}$ & 4.7 & 2.8 & 3.48 & 5.0 & 2.7 & 2.8 & 3.00 & 3.70 & 2.8 \\
\hline $\mathrm{K}_{2} \mathrm{O}$ & 2.8 & 2.1 & 3.75 & 2.7 & 6.0 & 5.1 & 2.50 & 4.00 & 2.7 \\
\hline $\mathrm{H}_{2}^{2} \mathrm{O}^{+}$ & 1.3 & 0.51 & 1.76 & 0.54 & 0.49 & 0.33 & 0.39 & 0.26 & 0.60 \\
\hline $\mathrm{H}_{2}^{2} \mathrm{O}^{-}$ & 0.34 & 0.15 & 0.34 & 0.27 & 0.10 & 0.30 & 0.15 & 0.13 & 0.26 \\
\hline $\mathrm{TiO}_{2}^{2}$ & 0.52 & 1.0 & 1.38 & 0.49 & 1.2 & 1.2 & 0.99 & 1.04 & 1.1 \\
\hline & 0.26 & 0.96 & 1.14 & 0.30 & 1.4 & 1.6 & 0.84 & 0.72 & 0.81 \\
\hline $\mathrm{MnO}$ & 0.07 & 0.18 & 0.135 & 0.08 & 0.15 & 0.16 & 0.14 & 0.084 & 0.16 \\
\hline $\mathrm{CO}_{2}$ & 0.52 & $<.05$ & 0.28 & $<.05$ & $<.05$ & $<.05$ & 0.15 & 0.16 & $<.05$ \\
\hline Total & 100.0 & 99.7 & 99.17 & 100.1 & 99.9 & 99.7 & 99.31 & 99.49 & 99.9 \\
\hline
\end{tabular}

CIPW norms (weight percent)

\begin{tabular}{|c|c|c|c|c|c|c|c|c|c|}
\hline Q & 15.53 & 0.00 & 0.00 & 12.74 & 0.00 & 0.00 & 0.00 & 2.84 & 0.00 \\
\hline or & 16.91 & 12.53 & 22.90 & 16.07 & 35.69 & 30.42 & 14.98 & 23.89 & 16.10 \\
\hline ab & 40.64 & 18.23 & 17.79 & 42.62 & 22.99 & 5.47 & 16.51 & 31.64 & 20.47 \\
\hline an & 16.52 & 18.52 & 10.17 & 16.91 & 8.14 & 4.61 & 16.99 & 12.48 & 15.90 \\
\hline C & 0.83 & 0.00 & 0.00 & 0.00 & 0.00 & 0.00 & 0.00 & 0.00 & 0.00 \\
\hline ne & 0.00 & 3.08 & 6.84 & 0.00 & 0.00 & 10.00 & 5.00 & 0.00 & 1.87 \\
\hline $\mathrm{di}$ & 0.00 & 18.96 & 23.02 & 1.67 & 13.20 & 21.44 & 19.52 & 9.07 & 20.66 \\
\hline ol & 0.00 & 20.66 & 9.37 & 0.00 & 8.03 & 17.72 & 19.28 & 0.00 & 16.89 \\
\hline hy & 4.95 & 0.00 & 0.00 & 5.40 & 2.15 & 0.00 & 0.00 & 12.65 & 0.00 \\
\hline $\mathrm{mt}$ & 2.99 & 3.66 & 4.32 & 2.91 & 3.94 & 3.95 & 3.66 & 3.72 & 3.81 \\
\hline il & 1.01 & 1.92 & 2.71 & 0.94 & 2.29 & 2.30 & 1.91 & 2.00 & 2.11 \\
\hline hem & 0.00 & 0.00 & 0.00 & 0.00 & 0.00 & 0.00 & 0.00 & 0.00 & 0.00 \\
\hline ap & 0.63 & 2.30 & 2.79 & 0.72 & 3.34 & 3.83 & 2.02 & 1.72 & 1.72 \\
\hline Total & 100.01 & 99.86 & 99.91 & 99.98 & 99.77 & 99.74 & 99.87 & 100.01 & 99.75 \\
\hline
\end{tabular}


Table 4. Some features of mapped and sampled granitic units

[-, no data]

\begin{tabular}{|c|c|c|c|}
\hline Pluton or mass & $\begin{array}{l}\text { Area, } \\
\left(\mathrm{km}^{2}\right)\end{array}$ & $\begin{array}{c}\text { Average } \\
\text { specific gravity }\end{array}$ & $\begin{array}{l}\text { Average magnetic } \\
\text { susceptibility }\end{array}$ \\
\hline Granite of Tehipite Dome & 72.4 & 2.65 & 1768 \\
\hline Granite of Alpine Creek & 5.6 & 2.65 & 1480 \\
\hline Granodiorite of White Divide & 89.7 & 2.65 & 2113 \\
\hline Cartridge Pass pluton & 9.6 & 2.67 & - \\
\hline Granite of Kennedy Lakes & 19.6 & 2.63 & 2192 \\
\hline Granodiorite of North Dome & 70.6 & 2.67 & 2425 \\
\hline Pyramid pluton & 32.1 & 2.73 & 5855 \\
\hline Granodiorite of Muro Blanco & 13.0 & 2.69 & 3100 \\
\hline Arrow pluton & 10.5 & 2.69 & 3000 \\
\hline Granite of Grand Dike & 2.4 & 2.62 & 7200 \\
\hline Granodiorite of Lightning Creek & 8.6 & 2.75 & 4300 \\
\hline Granoodiorite of Mitchell Peak & 9.7 & 2.73 & 4133 \\
\hline Granodiorite of Lookout Peak & 76.4 & 2.70 & 4605 \\
\hline Granite of North Mountain & 14.4 & 2.63 & 1230 \\
\hline Granodiorite of Sugarloaf & 11.8 & 2.71 & 4600 \\
\hline Paradise Granodiorite & 117.0 & 2.68 & 3820 \\
\hline Granite of Dougherty Peak & 10.5 & 2.62 & - \\
\hline Granite of Tunemah Lake & 4.5 & 2.62 & - \\
\hline Granite of LeConte Canyon & 2.5 & 2.61 & - \\
\hline Granodiorite of Upper Blue Canyon & 0.7 & 2.67 & 一 \\
\hline Older sheared granodiorite & 4.5 & 2.69 & - \\
\hline Mafic plutonic rock & 2.8 & 2.80 & 一 \\
\hline Total granitic rock & 589.0 & & \\
\hline Average granitic rock (by area) & & 2.674 & \\
\hline Metamorphic rock & 24.7 & & \\
\hline Tertiary dacite & 5.6 & & . \\
\hline Tertiary basalt & 0.7 & & \\
\hline Total quadrangle & 620.0 & & \\
\hline
\end{tabular}

Table 5. Average chemical composition, in weight percent, of granitic rocks in the Marion Peak quadrangle

\begin{tabular}{ll}
\hline $\mathrm{SiO}_{2}$ & 69.2 \\
$\mathrm{Al}_{2} \mathrm{O}_{3}$ & 15.1 \\
$\mathrm{Fe}_{2} \mathrm{O}_{3}$ & 1.2 \\
$\mathrm{FeO}$ & 1.3 \\
$\mathrm{MgO}$ & 0.95 \\
$\mathrm{CaO}$ & 2.8 \\
$\mathrm{Na} O$ & 3.7 \\
$\mathrm{~K}_{2} \mathrm{O}$ & 3.9 \\
$\mathrm{H}_{2} \mathrm{O}^{+}$ & 0.38 \\
$\mathrm{H}_{2} \mathrm{O}^{-}$ & 0.90 \\
$\mathrm{TiO}_{2}$ & 0.38 \\
$\mathrm{P}_{2} \mathrm{O}_{5}$ & 0.16 \\
$\mathrm{MnO}^{2}$ & 0.1 \\
\cline { 2 - 2 } Total & 100.1 \\
\hline
\end{tabular}





\section{SELECTED SERIES OF U.S. GEOLOGICAL SURVEY PUBLICATIONS}

\section{Periodicals}

Earthquakes \& Volcanoes (issued bimonthly).

Preliminary Determination of Epicenters (issued monthly).

\section{Technical Books and Reports}

Professional Papers are mainly comprehensive scientific reports of wide and lasting interest and importance to professional scientists and engineers. Included are reports on the results of resource studies and of topographic, hydrologic, and geologic investigations. They also include collections of related papers addressing different aspects of a single scientific topic.

Bulletins contain significant data and interpretations that are of lasting scientific interest but are generally more limited in scope or geographic coverage than Professional Papers. They include the results of resource studies and of geologic and topographic investigations; as well as collections of short papers related to a specific topic.

Water-Supply Papers are comprehensive reports that present significant interpretive results of hydrologic investigations of wide interest to professional geologists, hydrologists, and engineers. The series covers investigations in all phases of hydrology, including hydrogeology, availability of water, quality of water, and use of water.

Circulars present administrative information or important scientific information of wide popular interest in a format designed for distribution at no cost to the public. Information is usually of short-term interest.

Water-Resources Investigations Reports are papers of an interpretive nature made available to the public outside the formal USGS publications series. Copies are reproduced on request unlike formal USGS publications, and they are also available for public inspection at depositories indicated in USGS catalogs.

Open-File Reports include unpublished manuscript reports, maps, and other material that are made available for public consultation at depositories. They are a nonpermanent form of publication that may be cited in other publications as sources of information.

\section{Maps}

Geologic Quadrangle Maps are multicolor geologic maps on topographic bases in $71 / 2$ - or 15 -minute quadrangle formats (scales mainly 1:24,000 or 1:62,500) showing bedrock, surficial, or engineering geology. Maps generally include brief texts; some maps include structure and columnar sections only.

Geophysical Investigations Maps are on topographic or planimetric bases at various scales; they show results of surveys using geophysical techniques, such as gravity, magnetic, seismic, or radioactivity, which reflect subsurface structures that are of economic or geologic significance. Many maps include correlations with the geology.

Miscellaneous Investigations Series Maps are on planimetric or topographic bases of regular and irregular areas at various scales; they present a wide variety of format and subject matter. The series also includes $71 / 2$-minute quadrangle photogeologic maps on planimetric bases which show geology as interpreted from aerial photographs. Series also includes maps of Mars and the Moon.
Coal Investigations Maps are geologic maps on topographic or planimetric bases at various scales showing bedrock or surficial geology, stratigraphy, and structural relations in certain coal-resource areas.

Oil and Gas Investigations Charts show stratigraphic information for certain oil and gas fields and other areas having petroleum potential.

Miscellaneous Field Studies Maps are multicolor or black-andwhite maps on topographic or planimetric bases on quadrangle or irregular areas at various scales. Pre-1971 maps show bedrock geology in relation to specific mining or mineral-deposit problems; post-1971 maps are primarily black-and-white maps on various subjects such as environmental studies or wildemess mineral investigations.

Hydrologic Investigations Atlases are multicolored or black-andwhite maps on topographic or planimetric bases presenting a wide range of geohydrologic data of both regular and irregular areas; principal scale is $1: 24,000$ and regional studies are at $1: 250,000$ scale or smaller.

\section{Catalogs}

Permanent catalogs, as well as some others, giving comprehensive listings of U.S. Geological Survey publications are available under the conditions indicated below from the U.S. Geological Survey, Books and Open-File Reports Section, Federal Center, Box 25425, Denver, CO 80225. (See latest Price and Availability List.)

"Publications of the Geological Survey, 1879-1961" may be purchased by mail and over the counter in paperback book form and as a set of microfiche.

"Publications of the Geological Survey, 1962- 1970" may be purchased by mail and over the counter in paperback book form and as a set of microfiche.

"Publications of the U.S. Geological Survey, 1971-1981" may be purchased by mail and over the counter in paperback book form (two volumes, publications listing and index) and as a set of microfiche.

Supplements for 1982, 1983, 1984, 1985, 1986, and for subsequent years since the last permanent catalog may be purchased by mail and over the counter in paperback book form.

State catalogs, "List of U.S. Geological Survey Geologic and Water-Supply Reports and Maps For (State)," may be purchased by mail and over the counter in paperback booklet form only.

"Price and Availability List of U.S. Geological Survey Publicathons," issued annually, is available free of charge in paperback booklet form only.

Selected coples of a monthly catalog "New Publications of the U.S. Geological Survey" available free of charge by mail or may be obtained over the counter in paperback booklet form only. Those wishing a free subscription to the monthly catalog "New Publications of the U.S. Geological Survey" should write to the U.S. Geological Survey, 582 National Center, Reston, VA 22092.

Note.--Prices of Government publications listed in older catalogs, announcements, and publications may be incorrect. Therefore, the prices charged may differ from the prices in catalogs, announcements, and publications. 


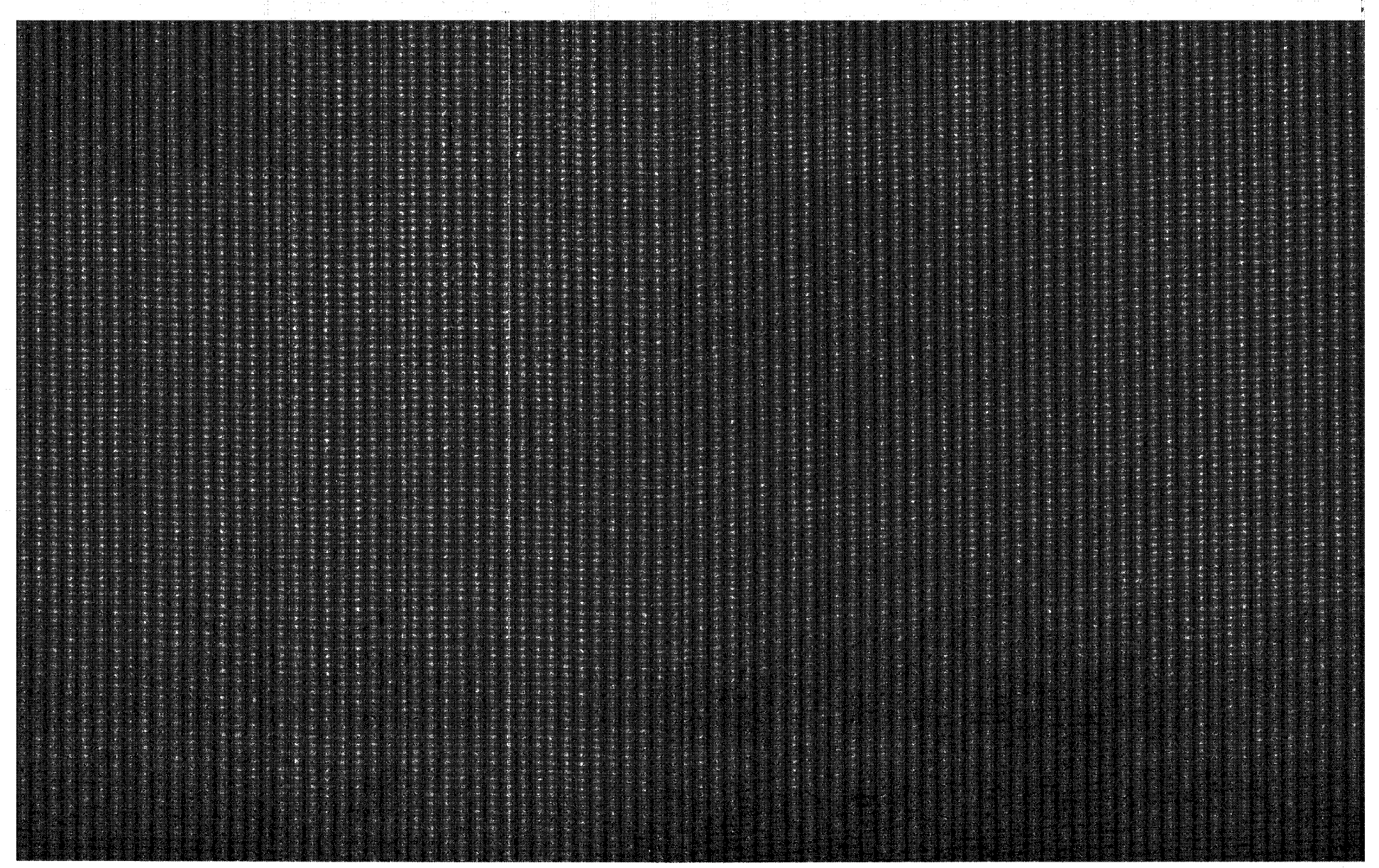

